\title{
Monomial ideals, edge ideals of hypergraphs, and their graded Betti numbers
}

\author{
Huy Tài Hà • Adam Van Tuyl
}

Received: 26 October 2006 / Accepted: 14 May 2007 / Published online: 7 July 2007

(C) Springer Science+Business Media, LLC 2007

\begin{abstract}
We use the correspondence between hypergraphs and their associated edge ideals to study the minimal graded free resolution of squarefree monomial ideals. The theme of this paper is to understand how the combinatorial structure of a hypergraph $\mathcal{H}$ appears within the resolution of its edge ideal $\mathcal{I}(\mathcal{H})$. We discuss when recursive formulas to compute the graded Betti numbers of $\mathcal{I}(\mathcal{H})$ in terms of its subhypergraphs can be obtained; these results generalize our previous work (Hà, H.T., Van Tuyl, A. in J. Algebra 309:405-425, 2007) on the edge ideals of simple graphs. We introduce a class of hypergraphs, which we call properly-connected, that naturally generalizes simple graphs from the point of view that distances between intersecting edges are "well behaved." For such a hypergraph $\mathcal{H}$ (and thus, for any simple graph), we give a lower bound for the regularity of $\mathcal{I}(\mathcal{H})$ via combinatorial information describing $\mathcal{H}$ and an upper bound for the regularity when $\mathcal{H}=G$ is a simple graph. We also introduce triangulated hypergraphs that are properly-connected hypergraphs generalizing chordal graphs. When $\mathcal{H}$ is a triangulated hypergraph, we explicitly compute the regularity of $\mathcal{I}(\mathcal{H})$ and show that the graded Betti numbers of $\mathcal{I}(\mathcal{H})$ are independent of the ground field. As a consequence, many known results about the graded Betti numbers of forests can now be extended to chordal graphs.
\end{abstract}

Keywords Hypergraphs · Chordal graphs · Monomial ideals · Graded resolutions · Regularity

Dedicated to Anthony V. Geramita on the occasion of his 65th birthday.

H.T. Hà (凶)

Department of Mathematics, Tulane University, 6823 St. Charles Ave., New Orleans, LA 70118,

USA

e-mail: tai@math.tulane.edu

\section{A. Van Tuyl}

Department of Mathematical Sciences, Lakehead University, , Thunder Bay, ON P7B 5E1, Canada e-mail: avantuyl@sleet.lakeheadu.ca 


\section{Introduction}

Let $\mathcal{X}=\left\{x_{1}, \ldots, x_{n}\right\}$ be a finite set, and let $\mathcal{E}=\left\{E_{1}, \ldots, E_{s}\right\}$ be a family of distinct subsets of $\mathcal{X}$. The pair $\mathcal{H}=(\mathcal{X}, \mathcal{E})$ is called a hypergraph if $E_{i} \neq \emptyset$ for each $i$. The elements of $\mathcal{X}$ are called the vertices, while the elements of $\mathcal{E}$ are called the edges of $\mathcal{H}$. A hypergraph $\mathcal{H}$ is simple if: (1) $\mathcal{H}$ has no loops, i.e., $|E| \geq 2$ for all $E \in \mathcal{E}$, and (2) $\mathcal{H}$ has no multiple edges, i.e., whenever $E_{i}, E_{j} \in \mathcal{E}$ and $E_{i} \subseteq E_{j}$, then $i=j$. A hypergraph generalizes the classical notion of a graph; a graph is a hypergraph for which every $E \in \mathcal{E}$ has cardinality two.

Let $k$ be a field. By identifying the vertex $x_{i}$ with the variable $x_{i}$ in the ring $R=k\left[x_{1}, \ldots, x_{n}\right]$, we can associate to every simple hypergraph $\mathcal{H}=(\mathcal{X}, \mathcal{E})$ the squarefree monomial ideal

$$
\mathcal{I}(\mathcal{H})=\left(\left\{x^{E}=\prod_{x \in E} x \mid E \in \mathcal{E}\right\}\right) \subseteq R=k\left[x_{1}, \ldots, x_{n}\right]
$$

We call the ideal $\mathcal{I}(\mathcal{H})$ the edge ideal of $\mathcal{H}$.

In this paper, we study the minimal graded free resolution of $\mathcal{I}(\mathcal{H})$. Since there is a natural bijection between the sets

$$
\left\{\begin{array}{c}
\text { simple hypergraphs } \mathcal{H}=(\mathcal{X}, \mathcal{E}) \\
\text { with } \mathcal{X}=\left\{x_{1}, \ldots, x_{n}\right\}
\end{array}\right\} \leftrightarrow\left\{\begin{array}{c}
\text { squarefree monomial } \\
\text { ideals } I \subseteq R=k\left[x_{1}, \ldots, x_{n}\right]
\end{array}\right\},
$$

we are in fact studying a fundamental problem in commutative algebra which asks for the minimal graded free resolution of a monomial ideal (for an introduction, see [24]). The edge ideal approach allows us to study this problem from a new angle; the standard approach is to use the Stanley-Reisner dictionary to associate to a squarefree monomial ideal $I$ a simplicial complex $\Delta$ where the generators of $I$ correspond to the minimal nonfaces of $\Delta$. Instead, we associate to $I$ a new combinatorial object, namely, a hypergraph. The theme of this work is to understand how the algebraic invariants of $I=\mathcal{I}(\mathcal{H})$ encoded in its minimal free resolution relate to the combinatorial properties of $\mathcal{H}$.

The edge ideal of a hypergraph was first introduced by Villarreal [31] in the special case that $\mathcal{H}=G$ is a simple graph. Subsequently, many people, including [1, 12, $13,15-18,27-30,32]$, have been working on a program to build a dictionary between the algebraic properties of $\mathcal{I}(G)$ and combinatorial structure of $G$. Of particular relevance to this paper, the minimal graded resolution of $\mathcal{I}(G)$ was investigated in $[5,7$, 9, 19-22, 26, 33] (see also [23] for a survey). In this paper, we extend some of these results to the hypergraph case, most notably, the results of [22], thereby extending our understanding of quadratic squarefree monomial ideals to arbitrary squarefree monomial ideals. At the same time, we also derive new results which, even when restricted to graphs, give new and interesting corollaries.

The edge ideal $\mathcal{I}(\mathcal{H})$ of an arbitrary hypergraph was first studied by Faridi [11] but from a slightly different perspective. Recall that $\Delta$ is a simplicial complex on the vertex set $\mathcal{X}$ if $\left\{x_{i}\right\} \in \Delta$ for all $i$, and if $F \in \Delta$, then all subsets of $F$ belong to $\Delta$. The facets of $\Delta$ are the maximal elements of $\Delta$ under inclusion. The facet ideal of $\Delta$ is then defined to be the ideal $\mathcal{I}(\Delta)=\left(\left\{x^{F}=\prod_{x \in F} x \mid F\right.\right.$ is a facet of $\left.\left.\Delta\right\}\right) \subseteq R$. Note, 
however, that if $\mathcal{F}(\Delta)=\left\{F_{1}, \ldots, F_{t}\right\}$ denotes the set of facets of $\Delta$, then $\mathcal{H}(\Delta)=$ $(\mathcal{X}, \mathcal{F}(\Delta))$ is a hypergraph. In fact, what Caboara, Faridi, and Selinger [3] call a facet complex is a hypergraph. It is immediate that $\mathcal{I}(\mathcal{H}(\Delta))=\mathcal{I}(\Delta)$. Conversely, given any hypergraph $\mathcal{H}=(\mathcal{X}, \mathcal{E})$, we can associate to $\mathcal{H}$ the simplicial complex $\Delta(\mathcal{H})=\left\{F \subseteq \mathcal{X} \mid F \subseteq E_{i}\right.$ for some $\left.E_{i} \in \mathcal{E}\right\}$. It is again easy to verify that $\mathcal{I}(\mathcal{H})=$ $\mathcal{I}(\Delta(\mathcal{H}))$.

One may therefore take the viewpoint that the generators of a squarefree monomial ideal correspond to either the edges of a hypergraph or the facets of a simplicial complex. In this paper, we have chosen to take the first option for at least two reasons: first, the language of hypergraphs is more natural to describe our results; and second, we only require the edge structure of the hypergraph and never make use of the simplicial complex structure. (A hypergraph point of view is also taken in the recent paper [15].) Of course, all our results could be reinterpreted as statements about the facet ideal of some simplicial complex.

The starting point of this paper is to determine how the splitting technique used in [22] to study the resolution of edge ideals of graphs can be extended to hypergraphs. Recall that Eliahou and Kervaire [8] call a monomial ideal I splittable if $I=J+K$ for two monomial ideas $J$ and $K$ such that the minimal generators of $J, K$, and $J \cap K$ satisfy a technical condition (see Definition 2.3 for the precise statement). When an ideal is splittable, the minimal resolutions (specifically the graded Betti numbers) of $I, J, K$, and $J \cap K$ are then related. Given a hypergraph $\mathcal{H}$, we therefore want to split $\mathcal{I}(\mathcal{H})$ so that the ideals $J, K$, and $J \cap K$ correspond to edge ideals of sub-hypergraphs of $\mathcal{H}$. This allows us to derive recursive-type formulas to relate the graded Betti numbers of $\mathcal{I}(\mathcal{H})$ to those of sub-hypergraphs of $\mathcal{H}$. These formulas provide a systematic approach to investigating algebraic invariants and properties of $\mathcal{I}(\mathcal{H})$.

We now summarize the results of this paper. In Sect. 3 we extend the notion of a splitting edge of a graph as defined in [22] to the hypergraph setting. Precisely, let $E$ be an edge of the hypergraph $\mathcal{H}$. If $\mathcal{H} \backslash E$ denotes the hypergraph with the edge $E$ removed, then it is clear that $\mathcal{I}(\mathcal{H})=\left(x^{E}\right)+\mathcal{I}(\mathcal{H} \backslash E)$. We call $E$ a splitting edge precisely when $\mathcal{I}(\mathcal{H})=\left(x^{E}\right)+\mathcal{I}(\mathcal{H} \backslash E)$ is a splitting of the ideal $\mathcal{I}(\mathcal{H})$. Our main result in Sect. 3 is the following classification of splitting edges, thus answering a question raised in [23].

Theorem 1.1 (Theorem 3.2) Let $\mathcal{H}$ be a hypergraph with two or more edges. Then an edge $E$ is a splitting edge of $\mathcal{H}$ if and only if there exists a vertex $z \in E$ such that

$$
\left(x^{E}\right) \cap \mathcal{I}(\mathcal{H} \backslash E) \subseteq\left(x^{E}\right) \cap \mathcal{I}(\mathcal{H} \backslash\{z\}) .
$$

Here, $\mathcal{H} \backslash\{z\}$ denotes the sub-hypergraph of $\mathcal{H}$ where every edge containing $z$ is removed.

To make use of our classification of splitting edges, we need to be able to describe the resolution of $J \cap K=\left(x^{E}\right) \cap \mathcal{I}(\mathcal{H} \backslash E)$. This resolution was described when $\mathcal{H}=$ $G$ is a simple graph in [23]. However, this is a difficult problem for an arbitrary $\mathcal{H}$. We are therefore interested in families of hypergraphs, which includes all simple graphs, where one can say something about $J \cap K$. 
In Sect. 4 we introduce one such family which we call properly-connected hypergraphs. A hypergraph $\mathcal{H}=(\mathcal{X}, \mathcal{E})$ is properly-connected if all its edges have the same cardinality and, furthermore, if $E, H \in \mathcal{E}$ with $E \cap H \neq \emptyset$, then the distance $\operatorname{dist}_{\mathcal{H}}(E, H)$ between $E$ and $H$, that is, the length of the shortest path between $E$ and $H$ in $\mathcal{H}$, is determined by $|E \cap H|$. It is easy to see that all simple graphs are properly-connected. In fact, a re-examination of the results of [22] reveals that the properly-connected property of graphs is an essential ingredient implicitly used in the proofs. A properly-connected hypergraph is in some sense a natural generalization of a simple graph.

When $\mathcal{H}$ is properly-connected, we can describe the resolution of $J \cap K$ in terms of edge ideals of sub-hypergraphs of $\mathcal{H}$. Therefore, for any splitting edge $E \in \mathcal{H}$, we can derive the following recursive-type formula for $\beta_{i, j}(\mathcal{I}(\mathcal{H}))$.

Theorem 1.2 (Theorem 4.16) Let $\mathcal{H}$ be a properly-connected hypergraph, and let $E$ be a splitting edge of $\mathcal{H}$. Suppose that $d=|E|, \mathcal{H}^{\prime}=\left\{H \in \mathcal{H} \mid \operatorname{dist}_{\mathcal{H}}(E, H) \geq d+1\right\}$, and $t=|N(E)|$, where

$$
N(E)=\bigcup_{\left\{H \in \mathcal{H} \mid \operatorname{dist}_{\mathcal{H}}(E, H)=1\right\}} H \backslash E .
$$

Then for all $i \geq 1$,

$$
\beta_{i, j}(\mathcal{I}(\mathcal{H}))=\beta_{i, j}(\mathcal{I}(\mathcal{H} \backslash E))+\sum_{l=0}^{i}\left(\begin{array}{l}
t \\
l
\end{array}\right) \beta_{i-1-l, j-d-l}\left(\mathcal{I}\left(\mathcal{H}^{\prime}\right)\right) .
$$

Here, $\beta_{-1, j}\left(\mathcal{I}\left(\mathcal{H}^{\prime}\right)\right)=1$ if $j=0$ and 0 if $j \neq 0$.

The sub-hypergraphs $\mathcal{H} \backslash E$ and $\mathcal{H}^{\prime}$ in Theorem 1.2 may fail to have splitting edges, thus preventing us from computing $\beta_{i, j}(\mathcal{I}(\mathcal{H})$ ) recursively. However, in [22] (see also $[19,20]$ in the case of forests), it is proved that when $\mathcal{H}$ is a hyperforest (i.e., a simplicial forest in the sense of [11]), then $\beta_{i, j}(\mathcal{I}(\mathcal{H}))$ can be computed recursively. The goal of Sect. 5 is to introduce a subclass of properly-connected hypergraphs, which we call triangulated hypergraphs, for which Theorem 1.2 can be used to completely resolve the graded Betti numbers of $\mathcal{I}(\mathcal{H})$ recursively. Triangulated hypergraphs generalize the notion of chordal graphs, which has attracted considerable attention lately (cf. $[12,13,17,18]$ ). In fact, triangulated graphs are precisely chordal graphs. As a consequence of Theorem 1.2, we also show that the graded Betti numbers of a triangulated hypergraph are independent of the characteristic of the ground field (Corollary 5.9). Restricted to simple graphs, we obtain the following interesting corollary, which extends a result of $[19,20]$ (that proved the result for forests).

Corollary 1.3 (Corollary 5.10) Suppose that $G$ is a chordal graph. Then the graded Betti numbers of $\mathcal{I}(G)$ are independent of the characteristic of the ground field and can be computed recursively.

In Sect. 6 we study $\operatorname{reg}(\mathcal{I}(\mathcal{H}))$, the Castelnuovo-Mumford regularity of $\mathcal{I}(\mathcal{H})$, when $\mathcal{H}$ is properly-connected. Again, the key idea we need here is the notion 
of distance between edges. We say that two edges $E, H \in \mathcal{H}$ are $t$-disjoint if $\operatorname{dist}_{\mathcal{H}}(E, H) \geq t$. When $\mathcal{H}$ is a properly-connected hypergraph and $d$ is the common cardinality of the edges, then $d$-disjoint edges are disjoint edges in the usual sense. We then show the following:

Theorem 1.4 Let $\mathcal{H}$ be a properly-connected hypergraph. Suppose that $d$ is the common cardinality of the edges in $\mathcal{H}$. Let $c$ be the maximal number of pairwise $(d+1)-$ disjoint edges of $\mathcal{H}$. Then

(i) (Theorem 6.5) $\operatorname{reg}(\mathcal{I}(\mathcal{H})) \geq(d-1) c+1$;

(ii) (Theorem 6.8) if $\mathcal{H}$ is also triangulated, then $\operatorname{reg}(\mathcal{I}(\mathcal{H}))=(d-1) c+1$.

By a matching of a hypergraph $\mathcal{H}$, we mean any subset $\mathcal{E}^{\prime} \subseteq \mathcal{E}$ of edges in $\mathcal{H}$ which are pairwise disjoint. The matching number of $\mathcal{H}$, denoted by $\alpha^{\prime}(\mathcal{H})$, is the largest size of a maximal matching of $\mathcal{H}$. For simple graphs, we also obtain a particularly nice upper bound for the regularity of $\mathcal{I}(G)$. This addresses a question J. Herzog had asked us.

Theorem 1.5 Theorem 6.7 Let $G$ be a finite simple graph. Then

$$
\operatorname{reg}(R / \mathcal{I}(G)) \leq \alpha^{\prime}(G)
$$

where $\alpha^{\prime}(G)$ is the matching number of $G$.

Using Theorem 1.5, we can compare the regularity and projective dimension of $\mathcal{I}(G)$ to those of $\mathcal{I}(G)^{\vee}$, the Alexander dual of $\mathcal{I}(G)$.

Theorem 1.6 (Theorem 6.14) Let $G$ be a simple graph.

(1) If $G$ is unmixed (i.e., all the minimal vertex covers have the same cardinality), then

$$
\begin{aligned}
& \operatorname{reg}(\mathcal{I}(G)) \leq \operatorname{ht} \mathcal{I}(G)+1 \leq \operatorname{reg}\left(\mathcal{I}(G)^{\vee}\right)+1 \quad \text { and } \\
& \operatorname{pdim}\left(\mathcal{I}(G)^{\vee}\right) \leq \operatorname{ht} \mathcal{I}(G) \leq \operatorname{pdim}(\mathcal{I}(G))+1
\end{aligned}
$$

(2) If $G$ is not unmixed, then

$$
\begin{aligned}
& \operatorname{reg}(\mathcal{I}(G)) \leq \operatorname{ht} \mathcal{I}(G)+1 \leq \operatorname{reg}\left(\mathcal{I}(G)^{\vee}\right) \quad \text { and } \\
& \operatorname{pdim}\left(\mathcal{I}(G)^{\vee}\right) \leq \operatorname{ht} \mathcal{I}(G) \leq \operatorname{pdim}(\mathcal{I}(G)) .
\end{aligned}
$$

When restricted to simple graphs, Theorem 1.4(ii) also gives an interesting corollary, which was first proved by Zheng [33] in the special case that $G$ was a forest.

Corollary 1.7 (Corollary 6.9) Let $G$ be a chordal graph. Then

$$
\operatorname{reg}(\mathcal{I}(G))=c+1
$$

where $c$ is the maximal number of 3-disjoint edges in $G$. 
Finally, in Sect. 7 we show that the first syzygy module of $\mathcal{I}(\mathcal{H})$ when $\mathcal{H}$ is properly-connected is generated by linear syzygies if and only if the diameter of the hypergraph $\mathcal{H}$ is small enough (Theorem 7.4). By the diameter we mean the maximum distance between any two edges of $\mathcal{H}$. This result can be seen as the first step towards generalizing Fröberg's result [14] characterizing graphs whose edge ideals have a linear resolution. As an interesting corollary, if $\mathcal{H}$ is a triangulated hypergraph, and if $\mathcal{I}(\mathcal{H})$ only has linear first syzygies, then the resolution of $\mathcal{I}(\mathcal{H})$ must in fact be linear (Corollary 7.6).

\section{Preliminaries}

We recall the relevant results concerning hypergraphs, resolutions, and splittable ideals.

\subsection{Hypergraphs and edge ideals}

Our reference for the hypergraph material is Berge [2].

Throughout this paper we assume that our hypergraphs $\mathcal{H}=(\mathcal{X}, \mathcal{E})$ are simple, i.e., $|E| \geq 2$ for all $E \in \mathcal{E}$, and there is no element of $\mathcal{E}$ which contains another. When there is no danger of confusion, we sometimes specify a hypergraph by describing only its set of edges.

If each $E \in \mathcal{E}$ has the same cardinality $d$, then we call $\mathcal{H}$ a $d$-uniform hypergraph. Note that a simple graph is a simple 2-uniform hypergraph. If $\mathcal{H}$ is $d$-uniform, then the associated simplicial complex $\Delta(\mathcal{H})$ is a pure simplicial complex, that is, all its facets have the same dimension.

If $E$ is an edge of a hypergraph $\mathcal{H}$, then by $\mathcal{H} \backslash E$ we denote the hypergraph formed by removing the edge $E$ from $\mathcal{H}$. Similarly, if $x$ is a vertex of $\mathcal{H}$, we write $\mathcal{H} \backslash\{x\}$ to denote the hypergraph formed by removing $x$ and all edges $E \in \mathcal{E}$ with the property that $x \in E$. Note that $x$ is an isolated vertex of $\mathcal{H} \backslash\{x\}$, or we can also consider the vertex set of $\mathcal{H} \backslash\{x\}$ to be $\mathcal{X} \backslash\{x\}$. If $\mathcal{Y} \subset \mathcal{X}$, then the induced hypergraph on $\mathcal{Y}$, denoted $\mathcal{H} \mathcal{Y}$, is the sub-hypergraph of $\mathcal{H}$ whose edge set is $\{E \in \mathcal{E} \mid E \subseteq \mathcal{Y}\}$. If there is no edge $E \in \mathcal{E}$ such that $E \subseteq \mathcal{Y}$, then we view $\mathcal{H}_{\mathcal{Y}}$ as the graph of the isolated vertices $\mathcal{Y}$.

The notion of distance between edges in a hypergraph will play a fundamental role in later discussions. We introduce the relevant definitions here.

Definition 2.1 A chain of length $n$ in $\mathcal{H}$ is a sequence $\left(E_{0}, x_{1}, E_{1}, \ldots, x_{n}, E_{n}\right)$ such that

(1) $x_{1}, \ldots, x_{n}$ are all distinct vertices of $\mathcal{H}$,

(2) $E_{0}, \ldots, E_{n}$ are all distinct edges of $\mathcal{H}$, and

(3) $x_{1} \in E_{0}, x_{n} \in E_{n}$, and $x_{k}, x_{k+1} \in E_{k}$ for each $k=1, \ldots, n-1$.

We sometimes denote the chain by $\left(E_{0}, \ldots, E_{n}\right)$ if the vertices in the chain are not being investigated. Note that (3) implies that $E_{i} \cap E_{i+1} \neq \varnothing$ for $i=0, \ldots, n-1$. If $E$ and $E^{\prime}$ are two edges, then $E$ and $E^{\prime}$ are connected if there exists a chain 
$\left(E_{0}, \ldots, E_{n}\right)$ such that $E=E_{0}$ and $E^{\prime}=E_{n}$. If $|E| \geq\left|E^{\prime}\right|$, then the chain connecting $E$ to $E^{\prime}$ is a proper chain if $\left|E_{i} \cap E_{i+1}\right|=\left|E_{i+1}\right|-1$ for all $i=0, \ldots, n-1$. The (proper) chain is an (proper) irredundant chain of length $n$ if no proper subsequence is a (proper) chain from $E$ to $E^{\prime}$.

Definition 2.2 If $E$ and $E^{\prime}$ are two edges of a hypergraph $\mathcal{H}$ with $|E| \geq\left|E^{\prime}\right|$, then we define the distance between $E$ and $E^{\prime}$, denoted by $\operatorname{dist}_{\mathcal{H}}\left(E, E^{\prime}\right)$, to be

$$
\operatorname{dist}_{\mathcal{H}}\left(E, E^{\prime}\right)=\min \left\{\ell \mid\left(E=E_{0}, \ldots, E_{\ell}=E^{\prime}\right) \text { is a proper irredundant chain }\right\} .
$$

If no proper irredundant chain between the two edges exists, we set $\operatorname{dist}_{\mathcal{H}}\left(E, E^{\prime}\right)=$ $\infty$.

As in the introduction, the edge ideal of $\mathcal{H}=(\mathcal{X}, \mathcal{E})$ is the squarefree monomial ideal

$$
\mathcal{I}(\mathcal{H})=\left(\left\{x^{E}=\prod_{x \in E} x \mid E \in \mathcal{E}\right\}\right) \subseteq R=k\left[x_{1}, \ldots, x_{n}\right]
$$

We often abuse notation and write $x^{E}$ for both the edge $E$ and the corresponding monomial.

\subsection{Resolutions and splittable ideals}

Let $M$ be a graded $R$-module where $R=k\left[x_{1}, \ldots, x_{n}\right]$. Associated to $M$ is a minimal graded free resolution of the form

$$
\begin{aligned}
0 & \rightarrow \bigoplus_{j} R(-j)^{\beta_{l, j}(M)} \rightarrow \bigoplus_{j} R(-j)^{\beta_{l-1, j}(M)} \rightarrow \cdots \rightarrow \bigoplus_{j} R(-j)^{\beta_{0, j}(M)} \\
& \rightarrow M \rightarrow 0
\end{aligned}
$$

where $l \leq n$ and $R(-j)$ is the $R$-module obtained by shifting the degrees of $R$ by $j$. The number $\beta_{i, j}(M)$, the $i j$ th graded Betti number of $M$, equals the number of minimal generators of degree $j$ in the $i$ th syzygy module of $M$.

Of particular interest are the following invariants which measure the "size" of the minimal graded free resolution of $I$. The regularity of $I$, denoted $\operatorname{reg}(I)$, is defined by

$$
\operatorname{reg}(I):=\max \left\{j-i \mid \beta_{i, j}(I) \neq 0\right\} .
$$

The projective dimension of $I$, denoted $\operatorname{pdim}(I)$, is defined to be

$$
\operatorname{pdim}(I):=\max \left\{i \mid \beta_{i, j}(I) \neq 0\right\} .
$$

An ideal $I$ generated by elements of degree $d$ is said to have a linear resolution if $\beta_{i, j}(I)=0$ for all $j \neq i+d$.

We now recall some results concerning splittable ideals. We use $\mathcal{G}(I)$ to denote the unique minimal set of generators of a monomial ideal $I$. 
Definition 2.3 (see [8]) A monomial ideal $I$ is splittable if $I$ is the sum of two nonzero monomial ideals $J$ and $K$, that is, $I=J+K$, such that

(1) $\mathcal{G}(I)$ is the disjoint union of $\mathcal{G}(J)$ and $\mathcal{G}(K)$;

(2) there is a splitting function

$$
\begin{aligned}
\mathcal{G}(J \cap K) & \rightarrow \mathcal{G}(J) \times \mathcal{G}(K), \\
w & \mapsto(\phi(w), \psi(w))
\end{aligned}
$$

satisfying

(a) for all $w \in \mathcal{G}(J \cap K), \quad w=\operatorname{lcm}(\phi(w), \psi(w))$,

(b) for every subset $S \subset \mathcal{G}(J \cap K)$, both $\operatorname{lcm}(\phi(S))$ and $\operatorname{lcm}(\psi(S))$ strictly divide $\operatorname{lcm}(S)$.

If $J$ and $K$ satisfy the above properties, then we say that $I=J+K$ is a splitting of $I$.

When $I=J+K$ is a splitting, then there is a relation between $\beta_{i, j}(I)$ and the graded Betti numbers of the "smaller" ideals. This relation was first observed for the total Betti numbers by Eliahou and Kervaire [8] and extended to the graded case by Fatabbi [10].

Theorem 2.4 Suppose that I is a splittable monomial ideal with splitting $I=J+K$. Then

$$
\beta_{i, j}(I)=\beta_{i, j}(J)+\beta_{i, j}(K)+\beta_{i-1, j}(J \cap K) \quad \text { for all } i, j \geq 0,
$$

where $\beta_{i-1, j}(J \cap K)=0$ if $i=0$.

When $I$ is a splittable ideal, Theorem 2.4 gives us the following corollary.

Corollary 2.5 If I is a splittable monomial ideal with splitting $I=J+K$, then

(i) $\operatorname{reg}(I)=\max \{\operatorname{reg}(J), \operatorname{reg}(K), \operatorname{reg}(J \cap K)-1\}$,

(ii) $\operatorname{pdim}(I)=\max \{\operatorname{pdim}(J), \operatorname{pdim}(K), \operatorname{pdim}(J \cap K)+1\}$.

Our goal is to study the numbers $\beta_{i, j}(\mathcal{I}(\mathcal{H}))$. From the definition of $\mathcal{I}(\mathcal{H})$ it directly follows that $\beta_{0, j}(\mathcal{I}(\mathcal{H}))$ is simply the number of edges $E \in \mathcal{H}$ with $|E|=j$. We can therefore restrict ourselves to investigating the numbers $\beta_{i, j}(\mathcal{I}(\mathcal{H}))$ with $i \geq 1$. When $\mathcal{H}$ is a $d$-uniform hypergraph, the following result implies that we only need to consider a finite range of values of $j$ for each $i$.

Theorem 2.6 Suppose that $\mathcal{H}$ is a d-uniform hypergraph. If $\beta_{i, j}(\mathcal{I}(\mathcal{H})) \neq 0$, then $i+d \leq j \leq \min \{n, d(i+1)\}$.

Proof Since $\mathcal{H}$ is a $d$-uniform hypergraph, $\mathcal{I}(\mathcal{H})$ is generated by monomials of degree $d$. So, $\beta_{i, j}(\mathcal{I}(\mathcal{H}))=0$ for $j<i+d$, thus giving us the lower bound. For the upper bound, the Taylor resolution implies that $\beta_{i, j}(\mathcal{I}(\mathcal{H}))=0$ if $j>d(i+1)$. On the other hand, Hochster's formula implies that $\beta_{i, j}(\mathcal{I}(\mathcal{H}))=0$ if $j>n$. The conclusion now follows. 


\section{Splitting edges}

Let $I$ be any squarefree monomial ideal, and suppose that $\mathcal{H}$ is the hypergraph associated to $I$, i.e., $I=\mathcal{I}(\mathcal{H})$. We would like to find splittings of $I$ so that we can make use of Theorem 2.4. In this section we describe one possible splitting of $\mathcal{I}(\mathcal{H})$.

One of the simplest ways to partition $\mathcal{G}(I)$ is to pick any $m \in \mathcal{G}(I)$ and set $\mathcal{G}(J)=$ $\{m\}$ and $\mathcal{G}(K)=\mathcal{G}(I) \backslash\{m\}$. Note that this is equivalent to picking any edge $E$ of $\mathcal{H}$ and setting

$$
J=\left(x^{E}\right) \quad \text { and } \quad K=\mathcal{I}(\mathcal{H} \backslash E) .
$$

It is immediate that $I=\mathcal{I}(\mathcal{H})=J+K$, and furthermore, $J$ and $K$ satisfy condition (1) of Definition 2.3. However, for an arbitrary edge $E, J$ and $K$ may fail to satisfy condition (2) of Definition 2.3. If $E$ is chosen so that $J$ and $K$ satisfy this condition, then we give this edge the following name.

Definition 3.1 Let $\mathcal{H}$ be a hypergraph. An edge $E$ is a splitting edge of $\mathcal{H}$ if

$$
\mathcal{I}(\mathcal{H})=\left(x^{E}\right)+\mathcal{I}(\mathcal{H} \backslash E)
$$

is a splitting of $\mathcal{I}(\mathcal{H})$.

To make use of Theorem 2.4, one would therefore like a means to identify the splitting edges of a hypergraph. The main result of this section is the following theorem which provides a classification of the splitting edges of a hypergraph. This theorem answers Question 5.4.2 of [23] which asked the equivalent question of what facet could be a splitting facet of simplicial complex.

Theorem 3.2 Let $\mathcal{H}$ be a hypergraph with two or more edges. Then an edge $E$ is a splitting edge of $\mathcal{H}$ if and only if there exists a vertex $z \in E$ such that

$$
\left(x^{E}\right) \cap \mathcal{I}(\mathcal{H} \backslash E) \subseteq\left(x^{E}\right) \cap \mathcal{I}(\mathcal{H} \backslash\{z\})
$$

Proof Let $E$ be an edge of $\mathcal{H}$, and set $J=\left(x^{E}\right)$ and $K=\mathcal{I}(\mathcal{H} \backslash E)$. To prove the "only if" direction, we prove the contrapositive. So, suppose that for every vertex $z \in E$, we have

$$
\left(x^{E}\right) \cap \mathcal{I}(\mathcal{H} \backslash E) \nsubseteq\left(x^{E}\right) \cap \mathcal{I}(\mathcal{H} \backslash\{z\}) .
$$

Thus, for each $z \in E$, there exists a minimal generator $x^{L_{z}}$ of $J \cap K$ such that $x^{L_{z}} \notin$ $\left(x^{E}\right) \cap \mathcal{I}(\mathcal{H} \backslash\{z\})$. Set $S=\left\{x^{L_{z}} \mid z \in E\right\} \subseteq \mathcal{G}(J \cap K)$.

We will now show that no splitting function can exist. Suppose that there is a splitting function $s: \mathcal{G}(J \cap K) \rightarrow \mathcal{G}(J) \times \mathcal{G}(K)$ given by $s(w)=(\phi(w), \varphi(w))$. Then, since $J=\left(x^{E}\right)$, for each $x^{L_{z}} \in S$, we have $\phi\left(x^{L_{z}}\right)=x^{E}$. For each $z \in E$, let $x^{G_{z}}=\varphi\left(x^{L_{z}}\right) \in \mathcal{G}(K)$. So $G_{z}$ is an edge of $\mathcal{H}$, and $\operatorname{lcm}\left(x^{E}, x^{G_{z}}\right)=x^{E \cup G_{z}}=x^{L_{z}}$.

We claim that for each $z \in E$, we have $z \in G_{z}$. Indeed, if $z^{\prime} \notin G_{z^{\prime}}$ for some $z^{\prime} \in E$, then $G_{z^{\prime}}$ is an edge of $\mathcal{H} \backslash\left\{z^{\prime}\right\}$. But then $x^{L z^{\prime}}=\operatorname{lcm}\left(x^{E}, x^{G_{z^{\prime}}}\right)=x^{E \cup G_{z^{\prime}}}$ is an element of $\left(x^{E}\right) \cap \mathcal{I}\left(\mathcal{H} \backslash\left\{z^{\prime}\right\}\right)$, a contradiction to the choice of $x^{L z^{\prime}}$. 
Now, since $z \in G_{z}$ for each $z \in E$, we have

$$
\begin{aligned}
\operatorname{lcm}(\varphi(S)) & =\operatorname{lcm}\left(\left\{x^{G_{z}} \mid z \in E\right\}\right)=x^{\cup_{z \in E} G_{z}}=x^{\left(\cup_{z \in E} G_{z}\right) \cup E}=x^{\cup_{z \in E}\left(G_{z} \cup E\right)} \\
& =x^{\cup_{z \in E} L_{z}}=\operatorname{lcm}\left(\left\{x^{L_{z}} \mid z \in E\right\}\right)=\operatorname{lcm}(S) .
\end{aligned}
$$

But this contradicts the fact that we have a splitting function. This proves the "only if" direction.

Conversely, suppose that there exists a vertex $z$ of $E$ such that

$$
\left(x^{E}\right) \cap \mathcal{I}(\mathcal{H} \backslash E) \subseteq\left(x^{E}\right) \cap \mathcal{I}(\mathcal{H} \backslash\{z\})
$$

This implies that $\mathcal{G}(J \cap K) \subseteq\left\{x^{E \cup H} \mid H \in \mathcal{H} \backslash\{z\}\right\}$. We will construct a splitting function $s=(\phi, \varphi): \mathcal{G}(J \cap K) \rightarrow \mathcal{G}(J) \times \mathcal{G}(K)$ which satisfies the conditions of Definition 2.3. For any $x^{L} \in \mathcal{G}(J \cap K)$, we define $\phi\left(x^{L}\right)=x^{E} \in \mathcal{G}(J)$. For each $x^{L} \in \mathcal{G}(J \cap K), \varphi\left(x^{L}\right)$ is defined as follows: by our hypothesis, we have $L \in\{E \cup$ $H \mid H \in \mathcal{H} \backslash\{z\}\}$. Thus, $\mathbb{A}=\{H \in \mathcal{H} \backslash\{z\} \mid L=E \cup H\}$ is not the empty set. We consider $\mathcal{X}$ as a set of alphabets (in some order of its elements) and identify each element of $\mathbb{A}$ with the word formed by its vertices (in increasing order). Let $G_{L}$ be the unique maximal element of $\mathbb{A}$ with respect to the lexicographic word ordering (which is a total order). Observe that, by construction, $z \notin G_{L}$ and $E \cup G_{L}=L$. Define $\varphi\left(x^{L}\right)=x^{G_{L}}$.

It is easy to see that $s=(\phi, \varphi)$ is a well-defined function on $\mathcal{G}(J \cap K)$ and that condition (a) of Definition 2.3 is satisfied. To show that condition (b) of Definition 2.3 is satisfied, we observe that for any $x^{L} \in \mathcal{G}(J \cap K)$, by construction, $z$ does not divide $\varphi\left(x^{L}\right)$. Observe further that for any subset $S \subseteq \mathcal{G}(J \cap K), z$ divides $x^{E}$ which strictly divides $\operatorname{lcm}(S)$. Thus, since $\operatorname{lcm}(\phi(S))=x^{E}$ and since $z$ does not divide $\operatorname{lcm}(\varphi(S))$, we must have that $\operatorname{lcm}(\phi(S))$ and $\operatorname{lcm}(\varphi(S))$ both strictly divide $\operatorname{lcm}(S)$. The "if" direction is proved.

Remark 3.3 Theorem 3.2 can be reinterpreted as describing when a squarefree monomial ideal $I=\left(m_{1}, \ldots, m_{s}\right)$ in $R=k\left[x_{1}, \ldots, x_{n}\right]$ has a splitting $I=\left(m_{i}\right)+$ $\left(m_{1}, \ldots, \hat{m}_{i}, \ldots, m_{s}\right)$ for some $i$. Precisely, $I=\left(m_{i}\right)+\left(m_{1}, \ldots, \hat{m}_{i}, \ldots, m_{s}\right)$ is a splitting if and only if there exists a variable $x_{j}$ such that $x_{j} \mid m_{i}$ and $\left(m_{i}\right) \cap$ $\left(m_{1}, \ldots, \hat{m}_{i}, \ldots, m_{s}\right) \subseteq\left(m_{i}\right) \cap I^{\prime} R$, where by $I^{\prime} R$ we mean the ideal $I^{\prime}=I \cap$ $k\left[x_{1}, \ldots, \hat{x}_{j}, \ldots, x_{n}\right]$, but viewed as an ideal of $R$. The result follows from the fact that $\mathcal{I}\left(\mathcal{H} \backslash\left\{x_{j}\right\}\right)=I^{\prime} R$. This reformulation nicely illustrates that in some cases the hypergraph point of view is conceptually easier (at least to us) to grasp.

Example 3.4 The following example illustrates that a hypergraph may not have a splitting edge. Let $\mathcal{H}$ be the hypergraph on vertex set $\mathcal{X}=\{a, b, c, d, e\}$ with edge set $\mathcal{E}=\{a b e$, ade, bce, $c d e\}$. The edge ideal is then $\mathcal{I}(\mathcal{H})=($ abe, ade, bce, cde $)$. By symmetry it suffices to show that any one of the edges is not a splitting edge. So, consider the edge $E=a b e$. Then

$$
\left(x^{E}\right) \cap \mathcal{I}(\mathcal{H} \backslash E)=(\text { abde }, \text { abce }, \text { abcde })=(\text { abde }, \text { abce }),
$$


while

$$
\begin{aligned}
& \left(x^{E}\right) \cap \mathcal{I}(\mathcal{H} \backslash\{a\})=(\text { abce }), \quad\left(x^{E}\right) \cap \mathcal{I}(\mathcal{H} \backslash\{b\})=(\text { abde }), \\
& \left(x^{E}\right) \cap \mathcal{I}(\mathcal{H} \backslash\{e\})=(0) .
\end{aligned}
$$

Thus, there is no vertex $z \in E$ with the property that $\left(x^{E}\right) \cap \mathcal{I}(\mathcal{H} \backslash E) \subseteq\left(x^{E}\right) \cap$ $\mathcal{I}(\mathcal{H} \backslash\{z\})$.

There is a nice class of edges of a simple hypergraph that are easy to identify and also have the property that they are splitting edges. We now define this class.

Definition 3.5 Let $\mathcal{H}$ be a simple hypergraph. An edge $E$ is a $v$-leaf if $E$ contains a free vertex, that is, $E$ contains a vertex $v \in \mathcal{X}$ such that $v$ does not belong to any other edge of $\mathcal{H}$.

Remark 3.6 If $\mathcal{H}=G$ is a simple graph, then $v$-leaves are precisely the leaves in the usual sense.

Corollary 3.7 Suppose that $E$ is a $v$-leaf of a hypergraph $\mathcal{H}$. Then $E$ is a splitting edge of $\mathcal{H}$.

Proof If $v$ is the free vertex in $E$, then $\mathcal{H} \backslash E=\mathcal{H} \backslash\{v\}$. Now apply Theorem 3.2.

Faridi [11] introduced the notion of a leaf for a simplicial complex $\Delta$. Precisely, a facet $F$ of $\Delta$ is a leaf if $F$ is the only facet of $\Delta$, or there exists a facet $G \neq F$ in $\Delta$ such that $F \cap F^{\prime} \subseteq F \cap G$ for all facets $F^{\prime} \neq F$ in $\Delta$. We can translate Faridi's definition into hypergraph language; we call the translated version of Faridi's leaf an $f$-leaf to distinguish it from a $v$-leaf.

Definition 3.8 An edge $E$ of a hypergraph $\mathcal{H}$ is an $f$-leaf if $E$ is the only edge of $\mathcal{H}$, or if there exists an edge $H$ of $\mathcal{H}$ such that $E \cap E^{\prime} \subseteq E \cap H$ for all edges $E^{\prime} \neq E$ of $\mathcal{H}$.

We introduce two types of hypertrees and hyperforests based upon the two notions of leaves.

Definition 3.9 A hypergraph $\mathcal{H}$ is a $v$-forest, respectively, $f$-forest, if every induced subgraph of $\mathcal{H}$, including $\mathcal{H}$ itself, contains a $v$-leaf, respectively, an $f$-leaf. If $\mathcal{H}$ is connected, we call $\mathcal{H}$ a $v$-tree, respectively, $f$-tree. When $\mathcal{H}$ is an $f$-forest, the associated simplicial complex $\Delta(\mathcal{H})$ is called a simplicial forest.

Notice that when $\mathcal{H}=G$ is a simple graph, the notions of $v$-leaf and $f$-leaf coincide. So, with simple graphs, the notions of a $v$-forest and an $f$-forest coincide with the usual notion of a forest. These definitions, however, are not equivalent in a general hypergraph, as illustrated below. 
Example 3.10 An $f$-leaf must always contain a free vertex (cf. [11, Remark 2.3]), thus every $f$-leaf is a $v$-leaf. However, a $v$-leaf need not be an $f$-leaf. For example, consider the hypergraph $\mathcal{H}$ on $\mathcal{X}=\{a, b, c, d, e, f\}$ with the edge set $\mathcal{E}=$ $\{a b f, b c d, d e f\}=\left\{E_{1}, E_{2}, E_{3}\right\}$. Each edge is a $v$-leaf, since each edge has a vertex not in the other two edges. However, $\mathcal{H}$ has no $f$-leaf. By symmetry, it is enough to show that $E_{1}=a b f$ cannot be an $f$-leaf. Indeed, $E_{1} \cap E_{2} \nsubseteq E_{1} \cap E_{3}$ and $E_{1} \cap E_{3} \nsubseteq E_{1} \cap E_{2}$.

The hypergraph $\mathcal{H}$ is an example of a $v$-tree, but $\mathcal{H}$ is not an $f$-tree, since $\mathcal{H}$ has no $f$-leaf, although all its induced subgraphs have an $f$-leaf.

Since an $f$-leaf is a $v$-leaf, Corollary 3.7 immediately gives:

Corollary 3.11 If $E$ is an $f$-leaf of a hypergraph $\mathcal{H}$, then $E$ is a splitting edge of $\mathcal{H}$.

\section{Properly-connected hypergraphs}

Given a hypergraph $\mathcal{H}$, we would like to express the numbers $\beta_{i, j}(\mathcal{I}(\mathcal{H})$ ) in terms of the graded Betti numbers of edge ideals associated to subgraphs of $\mathcal{H}$; this would lead to recursive-type formulas. When $E$ is a splitting edge of a hypergraph $\mathcal{H}$, Theorem 2.4 implies that $\beta_{i, j}(\mathcal{I}(\mathcal{H}))$ can be computed from the graded Betti numbers of the ideals $\left(x^{E}\right), \mathcal{I}(\mathcal{H} \backslash E)$, and $L=\left(x^{E}\right) \cap \mathcal{I}(\mathcal{H} \backslash E)$. The Betti numbers of $\left(x^{E}\right)$ are trivial to compute, while those of $\mathcal{I}(\mathcal{H} \backslash E)$ already correspond to the edge ideal of a sub-hypergraph of $\mathcal{H}$. Thus one only needs to relate the numbers $\beta_{i, j}(L)$ to the Betti numbers of an edge ideal of some other sub-hypergraph. For a general hypergraph, this appears to be a difficult problem.

The goal of this section is to introduce a family of $d$-uniform hypergraphs, which we call properly-connected, that among other things enables us to relate the graded Betti numbers of $L$ to those of an edge ideal associated to a sub-hypergraph of $\mathcal{H}$.

Definition 4.1 A $d$-uniform hypergraph $\mathcal{H}=(\mathcal{X}, \mathcal{E})$ is said to be properly-connected if for any two edges $E$ and $E^{\prime}$ of $\mathcal{H}$ with the property that $E \cap E^{\prime} \neq \emptyset$, we have

$$
\operatorname{dist}_{\mathcal{H}}\left(E, E^{\prime}\right)=d-\left|E \cap E^{\prime}\right| \text {. }
$$

Otherwise, we say that $\mathcal{H}$ is not properly-connected.

Remark 4.2 Our definition of properly-connected is similar to (but not equivalent to) what Zheng [33, Definition 3.14] called the intersection property for a simplicial complex. If $\Delta$ is a pure simplicial forest, then $\Delta$ has the intersection property if for any two facets $F, F^{\prime} \in \Delta$, the distance between $F$ and $F^{\prime}$ (defined in terms of the length of chain of between the two facets) is determined by $\left|F \cap F^{\prime}\right|$.

Example 4.3 Consider the 4-uniform hypergraph $\mathcal{H}$ with edge set

$$
\mathcal{E}=\left\{x_{1} x_{2} x_{3} x_{4}, x_{1} x_{2} x_{3} x_{7}, x_{1} x_{2} x_{6} x_{7}, x_{1} x_{5} x_{6} x_{7}, x_{1} x_{5} x_{6} x_{8}\right\} .
$$


There is a proper irredundant chain of length 4 from the edge $E=x_{1} x_{2} x_{3} x_{4}$ to $E^{\prime}=$ $x_{1} x_{5} x_{6} x_{8}$ (to form the chain, just take the edges as listed in $\mathcal{E}$ ). Furthermore, there is no shorter such chain. But $E$ and $E^{\prime}$ have a nonempty intersection. So $\mathcal{H}$ is not properly-connected, since $4=\operatorname{dist}_{\mathcal{H}}\left(E, E^{\prime}\right) \neq 4-\left|E \cap E^{\prime}\right|=3$. This hypergraph is not properly-connected.

Example 4.4 Every finite simple graph $G$ is properly-connected. To see this, note that a graph is clearly a 2-uniform hypergraph. If $E, E^{\prime}$ are two edges of $G$ such that $E \cap E^{\prime} \neq \varnothing$, then either $E$ and $E^{\prime}$ are the same edge, or $E$ and $E^{\prime}$ share exactly one vertex. In the first case, $\operatorname{dist}_{G}\left(E, E^{\prime}\right)=2-\left|E \cap E^{\prime}\right|=2-2=0$, while in the second case $\operatorname{dist}_{G}\left(E, E^{\prime}\right)=2-\left|E \cap E^{\prime}\right|=1$. So, in this sense, properly-connected hypergraphs generalize simple graphs.

Properly-connected hypergraphs are appealing combinatorial objects to study because within this family, the notions of $v$-leaf and $f$-leaf become equivalent. As well, splitting edges of properly-connected hypergraphs can be described combinatorially. We prove both of these assertions.

Theorem 4.5 Suppose that $\mathcal{H}$ is a d-uniform properly-connected hypergraph, and $E$ is an edge of $\mathcal{H}$. Then $E$ is a $v$-leaf if and only if $E$ is an $f$-leaf.

Proof Because we know that an $f$-leaf is a $v$-leaf, it suffices to prove the converse. If $\mathcal{H}$ has only one edge then we are done. So, suppose that $\mathcal{H}$ has at least two edges. Let $E$ be a $v$-leaf with free vertex $v$. Let $H$ be any edge of $\mathcal{H}$ with $H \cap E \neq \emptyset$. If there is no such $H$, then $E$ is automatically an $f$-leaf. Since $\mathcal{H}$ is properly-connected, there is a proper chain $E_{0}=E, E_{1}, \ldots, E_{k}=H$ from $E$ to $H$. Since $|E|=\left|E_{1}\right|=d$ and $\left|E \cap E_{1}\right|=d-1, E \cap E_{1}=E \backslash\{v\}$. To see that $E$ is an $f$-leaf, let $G$ be any other edge of $\mathcal{H}$. Then $E \cap G \subset E \backslash\{v\}=E \cap E_{1}$.

Let $E$ be an edge of a $d$-uniform properly-connected hypergraph $\mathcal{H}$. If $H$ is any edge of $\mathcal{H}$ with $\operatorname{dist}_{\mathcal{H}}(E, H)=1$, then $|H \backslash E|=1$, or in other words, $H \backslash E=\{z\}$ for some vertex $z$. Before classifying splitting edges, we introduce the following definition.

Definition 4.6 If $E$ is an edge of a $d$-uniform properly-connected hypergraph $\mathcal{H}$, then the vertex neighbor set of $E$ is the following subset of $\mathcal{X}$ :

$$
N(E)=\bigcup_{\left\{H \in \mathcal{H} \mid \operatorname{dist}_{\mathcal{H}}(E, H)=1\right\}} H \backslash E .
$$

Example 4.7 When $G$ is a finite simple graph, and $x$ is a vertex, then $N(x)$ denotes all the neighbors of $x$. If $E=\{u, v\}$ is any edge of $G$, then $N(E)=(N(u) \cup$ $N(v)) \backslash\{u, v\}$.

Theorem 4.8 Let $E$ be an edge of a d-uniform properly-connected hypergraph $\mathcal{H}$, and suppose that $N(E)=\left\{z_{1}, \ldots, z_{t}\right\}$. Then $E$ is a splitting edge if and only if there exists a vertex $z \in E$ such that $(E \backslash\{z\}) \cup\left\{z_{i}\right\} \in \mathcal{H}$ for each $z_{i} \in N(E)$. 
The proof of this theorem depends upon the following two lemmas.

Lemma 4.9 Let $\mathcal{H}$ be a $d$-uniform properly-connected hypergraph. Suppose that $E=E_{0}=\left\{x_{1}, \ldots, x_{d}\right\}$ and $E^{\prime}$ are edges in $\mathcal{H}$ with $\operatorname{dist}_{\mathcal{H}}\left(E, E^{\prime}\right)=t \leq d$. Then, after relabeling, there exist edges $E_{1}, \ldots, E_{t}$ such that $E_{i}=\left\{y_{1}, \ldots, y_{i}, x_{i+1}, \ldots, x_{d}\right\}$, $E_{t}=E^{\prime}$, and $y_{i} \notin E_{j}$ for all $j<i$.

Proof Since $\operatorname{dist}_{\mathcal{H}}\left(E, E^{\prime}\right)=t$, there must be a proper irredundant chain of edges $E_{0}=E, \ldots, E_{t}=E^{\prime}$. Since $E_{i}$ differs from $E_{i+1}$ by exactly one vertex, for each $i$, $\left|E \cap E_{i}\right| \geq d-i$, since at most one vertex changes at each stage. Since $\left(E_{0}, \ldots, E_{t}\right)$ is an irredundant chain and $\mathcal{H}$ is properly-connected, for $i<d$, we must have

$$
i=\operatorname{dist}_{\mathcal{H}}\left(E_{0}, E_{i}\right)=d-\left|E_{0} \cap E_{i}\right| .
$$

Hence, $\left|E_{0} \cap E_{i}\right|=d-i$ for any $i$ less than $d$ for which the expression makes sense. Moreover, if $i=t=d$, then $\operatorname{dist}_{\mathcal{H}}\left(E_{0}, E_{i}\right)=d$, and we have $E_{0} \cap E_{i}=\emptyset$. That is, $\left|E_{0} \cap E_{i}\right|=0=d-i$.

We will prove the result using induction on $i$. Let $E=E_{0}=\left\{x_{1}, \ldots, x_{d}\right\}$, and assume the vertices are labeled so that $x_{1} \notin E_{1}$. We know that $\left|E_{0} \cap E_{1}\right|=d-1$, which implies that $E_{1}=\left\{y_{1}, x_{2}, \ldots, x_{d}\right\}$ where $y_{1} \notin E_{0}$, thus proving the base case.

Now assume that $E_{0}, \ldots, E_{i}$ satisfy the claim, i.e., that $E_{i}=\left\{y_{1}, \ldots, y_{i}, x_{i+1}, \ldots\right.$, $x_{d}$ \} with $y_{i} \notin E_{j}$ for all $j<i$. We know that $\left|E_{i} \cap E_{i+1}\right|=d-1$, so that $E_{i+1}$ is constructed from $E_{i}$ by removing some vertex and adding a vertex that we will call $y_{i+1}$ which is not in $E_{i}$. First, we claim that the vertex that we remove from $E_{i}$ cannot be $y_{j}$ with $j \leq i$. If we were to replace some $y_{j}$ with a vertex $y_{i+1}$, then $\left|E_{0} \cap E_{i}\right|=d-i \leq\left|E_{0} \cap E_{i+1}\right|$, which contradicts our earlier assumption that $\left|E_{0} \cap E_{i+1}\right|=d-i-1$. So, we may assume that $y_{i+1}$ replaces $x_{i+1}$. If $y_{i+1}=x_{j}$ for some $j \leq i$, then $\left|E_{0} \cap E_{i+1}\right|=\left|E_{0} \cap E_{i}\right|$, which is a contradiction as before. Therefore, $y_{i+1} \notin E_{j}$ for any $j \leq i$.

Lemma 4.10 Let $E$ be any edge of a d-uniform properly-connected hypergraph $\mathcal{H}$. Then

$$
\begin{aligned}
\left(x^{E}\right) \cap \mathcal{I}(\mathcal{H} \backslash E)= & \left(\left\{\operatorname{lcm}\left(x^{E}, x^{H}\right) \mid H \in \mathcal{H} \text { and } \operatorname{dist}_{\mathcal{H}}(E, H)=1\right\}\right) \\
& +\left(\left\{\operatorname{lcm}\left(x^{E}, x^{H}\right) \mid H \in \mathcal{H} \text { and } \operatorname{dist}_{\mathcal{H}}(E, H) \geq d+1\right\}\right) .
\end{aligned}
$$

Proof Set

$$
\begin{aligned}
& A=\left(\left\{\operatorname{lcm}\left(x^{E}, x^{H}\right) \mid H \in \mathcal{H} \backslash E \text { and } \operatorname{dist}_{\mathcal{H}}(E, H) \leq d\right\}\right) \text { and } \\
& B=\left(\left\{\operatorname{lcm}\left(x^{E}, x^{H}\right) \mid H \in \mathcal{H} \text { and } \operatorname{dist}_{\mathcal{H}}(E, H) \geq d+1\right\}\right) .
\end{aligned}
$$

By definition $\left(x^{E}\right) \cap \mathcal{I}(\mathcal{H} \backslash E)=A+B$. Thus, if we set

$$
C=\left(\left\{\operatorname{lcm}\left(x^{E}, x^{H}\right) \mid H \in \mathcal{H} \text { and } \operatorname{dist}_{\mathcal{H}}(E, H)=1\right\}\right),
$$

then it suffices to show that $A=C$. Since $C \subseteq A$ is clear, we now show the reverse containment. 
Let $x^{E \cup H}=\operatorname{lcm}\left(x^{E}, x^{H}\right)$ be a generator of $A$, i.e., suppose that $H \in \mathcal{H} \backslash E$ and $t=\operatorname{dist}_{\mathcal{H}}(E, H) \leq d$. Note that we can assume that $2 \leq t \leq d$, since if $t=\operatorname{dist}_{\mathcal{H}}(E, H)=1$, then $x^{E \cup H} \in C$. So there exists a proper irredundant chain $E=H_{0}, H_{1}, H_{2}, \ldots, H_{t}=H$ whose length is minimal among all proper irredundant chains from $E$ to $H$.

Now if $E=\left\{x_{1}, \ldots, x_{d}\right\}$, then $H_{1}=\left\{x_{1}, \ldots, \hat{x}_{i}, \ldots, x_{d}, z\right\}$, where by $\hat{x}_{i}$ we mean that the vertex $x_{i}$ is removed, and $z$ is not one of $x_{1}, \ldots, x_{d}$. From this observation we have

$$
\operatorname{lcm}\left(x^{E}, x^{H_{1}}\right)=x^{E \cup\{z\}}=x^{E} z .
$$

Now $x^{E} z$ is a generator of $C$. To finish the proof, Lemma 4.9 implies that $z \in H_{i}$ for $i=2, \ldots, t$. Therefore, $\operatorname{lcm}\left(x^{E}, x^{H_{i}}\right)=x^{E \cup H_{i}}$ is divisible by $x^{E} z$ and thus is in $C$. In particular, $x^{E \cup H} \in C$.

Proof of Theorem 4.8 Suppose that $E$ is a splitting edge. By Theorem 3.2 there is a vertex $z \in E$ such that $\left(x^{E}\right) \cap \mathcal{I}(H \backslash E) \subseteq\left(x^{E}\right) \cap \mathcal{I}(H \backslash\{z\})$. Let $z_{i} \in N(E)$. We will show that $(E \backslash\{z\}) \cup\left\{z_{i}\right\}$ is an edge of $\mathcal{H} \backslash\{z\} \subseteq \mathcal{H}$. Since $z_{i} \in N(E)$, there exists an edge $H$ with $\operatorname{dist}_{\mathcal{H}}(E, H)=1$ such that $H \backslash E=\left\{z_{i}\right\}$. Thus, $x^{E \cup H}$ is a generator of $\left(x^{E}\right) \cap \mathcal{I}(\mathcal{H} \backslash E)$. We thus must have $x^{E \cup H} \in\left(x^{E}\right) \cap \mathcal{I}(\mathcal{H} \backslash\{z\})$. Hence there is an edge $H^{\prime} \in \mathcal{H} \backslash\{z\}$ such that $E \cup H=E \cup H^{\prime}$. Since $|E \cap H|=d-1$, we must have that $\left|E \cap H^{\prime}\right|=d-1$. Since $z \notin H^{\prime}$ and $z_{i} \notin E$, we must have $H^{\prime}=(E \backslash\{z\}) \cup\left\{z_{i}\right\}$. So, $(E \backslash\{z\}) \cup\left\{z_{i}\right\} \in \mathcal{H} \backslash\{z\}$, as desired.

Conversely, suppose that there exists a vertex $z \in E$ such that $(E \backslash\{z\}) \cup\left\{z_{i}\right\} \in \mathcal{H}$ for each $z_{i} \in N(E)$. Let $x^{L}$ be any minimal generator of $\left(x^{E}\right) \cap \mathcal{I}(\mathcal{H} \backslash E)$. By Lemma 4.10, we have $L=E \cup H$ with $\operatorname{dist}_{\mathcal{H}}(E, H)=1$ or $L=E \cup H$ with $\operatorname{dist}_{\mathcal{H}}(E, H) \geq d+1$. If $\operatorname{dist}_{\mathcal{H}}(E, H) \geq d+1$, then $z \notin H$ since $E \cap H=\emptyset$. So $H \in \mathcal{H} \backslash\{z\}$, and hence $x^{L} \in\left(x^{E}\right) \cap \mathcal{I}(\mathcal{H} \backslash\{z\})$. So, suppose that $L=E \cup H$ with $\operatorname{dist}_{\mathcal{H}}(E, H)=1$. Then, the exists $z_{i} \in N(E)$ such that $E \cup H=E \cup\left\{z_{i}\right\}$. By our hypothesis, the edge $E^{\prime}=(E \backslash\{z\}) \cup\left\{z_{i}\right\} \in \mathcal{H}$. But then $E^{\prime} \in \mathcal{H} \backslash\{z\}$. Furthermore, $L=E \cup H=E \cup E^{\prime}$. So $x^{L} \in\left(x^{E}\right) \cap \mathcal{I}(\mathcal{H} \backslash\{z\})$. We have now shown that $\left(x^{E}\right) \cap \mathcal{I}(\mathcal{H} \backslash E) \subseteq\left(x^{E}\right) \cap \mathcal{I}(\mathcal{H} \backslash\{z\})$, so by Theorem 3.2 the edge $E$ must be a splitting edge.

Example 4.11 We give an example of a 3-uniform properly-connected hypergraph which has a splitting edge that is not a $v$-leaf. Let $\mathcal{H}$ be the hypergraph with edge set

$$
\mathcal{E}=\left\{x_{1} x_{2} x_{3}, x_{1} x_{2} x_{4}, x_{1} x_{3} x_{5}, x_{2} x_{3} x_{4}, x_{2} x_{3} x_{5}, x_{3} x_{4} x_{5}\right\} .
$$

One can verify that $\mathcal{H}$ is properly connected by showing that $\operatorname{dist}_{\mathcal{H}}\left(E, E^{\prime}\right)=3-$ $\left|E \cap E^{\prime}\right|$ for every pair of edges in $\mathcal{E}$. Now $E=x_{1} x_{2} x_{3}$ is not a $v$-leaf, since it does not contain a free vertex. We can use Theorem 4.8 to verify that $E$ is a splitting edge. In this case $N(E)=\left\{x_{4}, x_{5}\right\}$, since the edges of distance one from $E$ are $\left\{x_{1} x_{2} x_{4}, x_{1} x_{3} x_{5}, x_{2} x_{3} x_{4}, x_{2} x_{3} x_{5}\right\}$. Then $E$ is a splitting edge, since $\left(E \backslash\left\{x_{1}\right\}\right) \cup\left\{x_{4}\right\}=$ $x_{2} x_{3} x_{4}$ and $\left(E \backslash\left\{x_{1}\right\}\right) \cup\left\{x_{5}\right\}=x_{2} x_{3} x_{5}$ are both edges of $\mathcal{H}$. Note that even when $E$ is a splitting edge, the graph $\mathcal{H} \backslash E$ may fail to be properly-connected. In this case, if we remove $E$ from $\mathcal{H}$, the resulting hypergraph fails to be properly-connected, since 
the edges $E_{1}=x_{1} x_{2} x_{4}$ and $E_{2}=x_{1} x_{3} x_{5}$ intersect at $x_{1}$, but there is no proper chain of length $2=3-\left|E_{1} \cap E_{2}\right|$ in $\mathcal{H} \backslash E$ between these two edges.

Notation 4.12 Suppose that $E$ is an edge of a $d$-uniform properly-connected hypergraph $\mathcal{H}$. For simplicity of notation, throughout the rest of the paper, when not specified, $\mathcal{H}^{\prime}$ refers to the sub-hypergraph

$$
\mathcal{H}^{\prime}=\left\{H \in \mathcal{H} \mid \operatorname{dist}_{\mathcal{H}}(E, H) \geq d+1\right\} .
$$

The following lemma tells us the properly-connected property is passed on to $\mathcal{H}^{\prime}$.

Lemma 4.13 If $E$ is an edge of a d-uniform properly-connected hypergraph $\mathcal{H}$, then $\mathcal{H}^{\prime}$ also is a d-uniform properly-connected hypergraph.

Proof Since it is clear that $\mathcal{H}^{\prime}$ is a $d$-uniform hypergraph, it suffices to show that $\mathcal{H}^{\prime}$ is properly connected. So, suppose that the edges $H, H^{\prime} \in \mathcal{H}^{\prime}$ have the property that $H \cap$ $H^{\prime} \neq \emptyset$. Since they also are edges of $\mathcal{H}$, there exists a chain $H=H_{0}, H_{1}, \ldots, H_{t}=$ $H^{\prime}$ in $\mathcal{H}$ such that $t=\operatorname{dist}_{\mathcal{H}}\left(H, H^{\prime}\right)=d-\left|H \cap H^{\prime}\right|$. If all the edges $H_{i}$ for $i=$ $1, \ldots, t-1$ are also in $\mathcal{H}^{\prime}$, then it is clear that $t=\operatorname{dist}_{\mathcal{H}^{\prime}}\left(H, H^{\prime}\right)=d-\left|H \cap H^{\prime}\right|$. So, suppose that there is an edge $H_{i}$ in the chain with $i \in\{1, \ldots, t-1\}$ and $H_{i} \notin \mathcal{H}^{\prime}$. Then $s=\operatorname{dist}_{\mathcal{H}}\left(E, H_{i}\right) \leq d$. Let $E=E_{0}, E_{1}, \ldots, E_{s}=H_{i}$ be the proper irredundant chain in $\mathcal{H}$ between $E$ and $H_{i}$. Then $\operatorname{dist}_{\mathcal{H}}\left(E_{1}, H_{i}\right)=s-1<d$. But this means that $\left|E_{1} \cap H_{i}\right| \neq \emptyset$. Let $x \in E_{1} \cap H_{i}$. By Lemma 4.9 the vertex $x$ must be in either $H$ or $H^{\prime}$. Without loss of generality, assume that $x \in H$. But then $\operatorname{dist}_{\mathcal{H}}\left(E_{1}, H\right)=$ $d-\left|H \cap E_{1}\right| \leq d-1$. But since $E$ is distance one from $E_{1}$, this means that there is a proper chain of length $d$ from $E$ to $H$, contradicting the fact that $H \in \mathcal{H}^{\prime}$.

As a byproduct of Lemma 4.10, we can rewrite $\left(x^{E}\right) \cap \mathcal{I}(\mathcal{H} \backslash E)$ in terms of the edge ideal of $\mathcal{H}^{\prime}$.

Corollary 4.14 Let $E$ be any edge of a d-uniform properly-connected hypergraph $\mathcal{H}$, and suppose that $N(E)=\left\{z_{1}, \ldots, z_{t}\right\}$. Then

$$
\left(x^{E}\right) \cap \mathcal{I}(\mathcal{H} \backslash E)=x^{E}\left(\left(z_{1}, \ldots, z_{t}\right)+\mathcal{I}\left(\mathcal{H}^{\prime}\right)\right) .
$$

Proof It is straightforward to verify that

$$
x^{E}\left(z_{1}, \ldots, z_{t}\right)=\left(\left\{\operatorname{lcm}\left(x^{E}, x^{H}\right) \mid H \in \mathcal{H} \text { and } \operatorname{dist}_{\mathcal{H}}(E, H)=1\right\}\right) .
$$

If $H \in \mathcal{H} \backslash E$ with $\operatorname{dist}_{\mathcal{H}}(E, H) \geq d+1$, then since $\mathcal{H}$ is properly-connected, $\mid E \cap$ $H \mid=\emptyset$. So

$$
x^{E} \mathcal{I}\left(\mathcal{H}^{\prime}\right)=\left(\left\{\operatorname{lcm}\left(x^{E}, x^{H}\right) \mid H \in \mathcal{H} \text { and } \operatorname{dist}_{\mathcal{H}}(E, H) \geq d+1\right\}\right) .
$$

The result now follows from Lemma 4.10.

When $E$ is an edge of a properly-connected hypergraph, we can also describe the graded Betti numbers of $\left(x^{E}\right) \cap \mathcal{I}(\mathcal{H} \backslash E)$ in terms of those of $\mathcal{I}\left(\mathcal{H}^{\prime}\right)$. 
Lemma 4.15 Let $E$ be any edge of a d-uniform properly-connected hypergraph $\mathcal{H}$. Set $t=|N(E)|$. Then

$$
\beta_{i-1, j}\left(\left(x^{E}\right) \cap \mathcal{I}(\mathcal{H} \backslash E)\right)=\sum_{l=0}^{i}\left(\begin{array}{l}
t \\
l
\end{array}\right) \beta_{i-1-l, j-d-l}\left(\mathcal{I}\left(\mathcal{H}^{\prime}\right)\right),
$$

where $\beta_{-1, j}\left(\mathcal{I}\left(\mathcal{H}^{\prime}\right)\right)=1$ if $j=0$ and 0 otherwise.

Proof If $N(E)=\left\{z_{1}, \ldots, z_{t}\right\}$, then by the previous corollary,

$$
\begin{aligned}
\beta_{i-1, j}\left(\left(x^{E}\right) \cap \mathcal{I}(\mathcal{H} \backslash E)\right) & =\beta_{i-1, j}\left(x^{E}\left(\left(z_{1}, \ldots, z_{t}\right)+\mathcal{I}\left(\mathcal{H}^{\prime}\right)\right)\right) \\
& =\beta_{i-1, j-d}\left(\left(z_{1}, \ldots, z_{t}\right)+\mathcal{I}\left(\mathcal{H}^{\prime}\right)\right) \\
& =\beta_{i, j-d}\left(R /\left(\left(z_{1}, \ldots, z_{t}\right)+\mathcal{I}\left(\mathcal{H}^{\prime}\right)\right)\right) .
\end{aligned}
$$

None of the generators of $\mathcal{I}\left(\mathcal{H}^{\prime}\right)$ are divisible by $z_{i}$ for $i=1, \ldots, t$. To see this, suppose that $x^{H} \in \mathcal{I}\left(\mathcal{H}^{\prime}\right)$ is divisible by some $z_{i}$, i.e., $z_{i}$ is a vertex of the edge $H$. Now there is an edge $H_{i}$ with $z_{i} \in H_{i}$ and $\operatorname{dist}_{\mathcal{H}}\left(E, H_{i}\right)=1$. Since $H \cap H_{i} \neq \emptyset$ and since $\mathcal{H}$ is properly-connected, $p=\operatorname{dist}_{\mathcal{H}}\left(H, H_{i}\right)=d-\left|H \cap H_{i}\right|<d$. So there is a proper irredundant chain $H_{i}=H_{0}^{\prime}, \ldots, H_{p}^{\prime}=H$. But then $E, H_{i}=H_{0}^{\prime}, \ldots, H_{p}^{\prime}=H$ forms a proper irredundant chain of length $p+1 \leq d$, and thus $\operatorname{dist}_{\mathcal{H}}(E, H) \leq d$, contradicting the fact that $\operatorname{dist}_{\mathcal{H}}(E, H) \geq d+1$.

We modify our notation and write $R=k\left[z_{1}, \ldots, z_{t}, x_{1}, \ldots, x_{s}\right]$, where $\left\{x_{1}, \ldots\right.$, $\left.x_{s}\right\}=\mathcal{X} \backslash N(E)$. Then

$$
R /\left(\left(z_{1}, \ldots, z_{t}\right)+\mathcal{I}\left(\mathcal{H}^{\prime}\right)\right) \cong R_{1} /\left(z_{1}, \ldots, z_{t}\right) \otimes_{k} R_{2} / \mathcal{I}\left(\mathcal{H}^{\prime}\right),
$$

where $R_{1}=k\left[z_{1}, \ldots, z_{t}\right]$ and $R_{2}=k\left[x_{1}, \ldots, x_{s}\right]$, and where we view $\mathcal{I}\left(\mathcal{H}^{\prime}\right)$ as an ideal of $R$ and as the ideal of $R_{2}$ generated by the same elements. By tensoring the resolutions of $R_{1} /\left(z_{1}, \ldots, z_{t}\right)$ and $R_{2} / \mathcal{I}\left(\mathcal{H}^{\prime}\right)$ together we get (see, for example, Lemma 2.1 and Corollary 2.2 of [20])

$$
\beta_{i, j-d}(R / L)=\sum_{l_{1}=0}^{i} \sum_{l_{2}=0}^{j-d} \beta_{l_{1}, l_{2}}\left(R_{1} /\left(z_{1}, \ldots, z_{t}\right)\right) \beta_{i-l_{1}, j-d-l_{2}}\left(R_{2} /\left(\mathcal{I}\left(\mathcal{H}^{\prime}\right)\right)\right)
$$

where $L=\left(z_{1}, \ldots, z_{t}\right)+\mathcal{I}\left(\mathcal{H}^{\prime}\right)$. Since $z_{1}, \ldots, z_{t}$ is a regular sequence on $R_{1}$,

$$
\beta_{l_{1}, l_{2}}\left(R_{1} /\left(z_{1}, \ldots, z_{t}\right)\right)= \begin{cases}0 & \text { if } l_{2} \neq l_{1} \\
\left(\begin{array}{l}
t \\
l
\end{array}\right) & \text { if } l=l_{2}=l_{1} .\end{cases}
$$

As a consequence, the previous expression reduces to

$$
\beta_{i, j-d}(R / L)=\sum_{l=0}^{i}\left(\begin{array}{l}
t \\
l
\end{array}\right) \beta_{i-l, j-d-l}\left(R_{2} / \mathcal{I}\left(\mathcal{H}^{\prime}\right)\right)
$$

We are now done, since

$$
\beta_{i-l, j-d-l}\left(R_{2} / \mathcal{I}\left(\mathcal{H}^{\prime}\right)\right)=\beta_{i-l, j-d-l}\left(R / \mathcal{I}\left(\mathcal{H}^{\prime}\right)\right)=\beta_{i-l-1, j-d-l}\left(\mathcal{I}\left(\mathcal{H}^{\prime}\right)\right)
$$


for all $l$ (where we adopt the convention that $\beta_{-1, j}\left(\mathcal{I}\left(\mathcal{H}^{\prime}\right)\right)=1$ if $j=0$ and 0 if $j \neq 0)$.

When $\mathcal{H}$ is a properly-connected hypergraph, we obtain the following recursivelike formula for $\beta_{i, j}(\mathcal{I}(\mathcal{H}))$. This result generalizes a similar result for simple graphs found in [22].

Theorem 4.16 Let $\mathcal{H}$ be a d-uniform properly-connected hypergraph, and let $E$ be a splitting edge of $\mathcal{H}$. Suppose that $\mathcal{H}^{\prime}=\left\{H \in \mathcal{H} \mid \operatorname{dist}_{\mathcal{H}}(E, H) \geq d+1\right\}$ and $t=$ $|N(E)|$. Then for all $i \geq 1$,

$$
\beta_{i, j}(\mathcal{I}(\mathcal{H}))=\beta_{i, j}(\mathcal{I}(\mathcal{H} \backslash E))+\sum_{l=0}^{i}\left(\begin{array}{l}
t \\
l
\end{array}\right) \beta_{i-1-l, j-d-l}\left(\mathcal{I}\left(\mathcal{H}^{\prime}\right)\right) .
$$

Here, $\beta_{-1, j}\left(\mathcal{I}\left(\mathcal{H}^{\prime}\right)\right)=1$ if $j=0$ and 0 if $j \neq 0$.

Proof Since $E$ is a splitting edge, by Theorem 2.4 we have

$$
\beta_{i, j}(\mathcal{I}(\mathcal{H}))=\beta_{i, j}\left(\left(x^{E}\right)\right)+\beta_{i, j}(\mathcal{I}(\mathcal{H} \backslash E))+\beta_{i-1, j}\left(\left(x^{E}\right) \cap \mathcal{I}(\mathcal{H} \backslash E)\right) .
$$

When $i \geq 1, \beta_{i, j}\left(\left(x^{E}\right)\right)=0$. Now substitute the formula of Lemma 4.15 into the last expression.

\section{Triangulated properly-connected hypergraphs}

If $\mathcal{H}$ is a properly-connected hypergraph with splitting edge $E$, the sub-hypergraphs $\mathcal{H} \backslash E$ and $\mathcal{H}^{\prime}$ in Theorem 4.16 may or may not have a splitting edge. In fact, $\mathcal{H} \backslash E$ may not even be a properly-connected hypergraph. These facts prevent us from using Theorem 4.16 to recursively compute $\beta_{i, j}(\mathcal{I}(\mathcal{H}))$ for any hypergraph. One is lead to ask if there is any subfamily of properly-connected hypergraphs for which the formula is recursive. In this section, we introduce one such family which generalizes the notion of a chordal graph. In [22] it was shown that hyperforests (i.e., a simplicial forest in the sense of [11]) is a family of hypergraphs for which the graded Betti numbers can be computed recursively. Since a hyperforest need not be properly-connected, the results of this section give a partial generalization of [22].

We begin by recalling the definition of a chordal graph.

Definition 5.1 A graph $G$ is called chordal if every cycle of length 4 or larger has a chord, that is, an edge joining two nonadjacent vertices in the cycle.

An alternative characterization for chordal graphs can be found in [25] (due to Dirac [6]). This characterization will prove more suitable when generalizing to properly-connected hypergraphs.

Theorem 5.2 A graph $G$ is chordal if and only if every induced subgraph of $G$ contains a vertex $v$ whose neighborhood $N(v)$ is a complete graph. 
In the above theorem, since $v$ is adjacent to every vertex in $N(v)$, we also have that the induced graph on $N(v) \cup\{v\}$ is also a complete graph. To extend this definition, we first introduce an analog of complete graphs.

Definition 5.3 The $d$-complete hypergraph of order $n$, denoted by $\mathcal{K}_{n}^{d}$, is the hypergraph consisting of all the $d$-subsets of the vertex set $\mathcal{X}$, where $|\mathcal{X}|=n$. When $d=2$, then $\mathcal{K}_{n}^{2}$ is the usual complete graph $\mathcal{K}_{n}$. When $n<d$, we consider $\mathcal{K}_{n}^{d}$ as the hypergraph with $n$ isolated vertices. If $n=0$, then $\mathcal{K}_{0}^{d}$ is the empty graph which we view as the $d$-complete hypergraph of order 0 .

Definition 5.4 Two distinct vertices $x, y \in \mathcal{X}$ are neighbors if there is an edge $E \in \mathcal{H}$ such that $x, y \in E$. For any vertex $x \in \mathcal{X}$, the neighborhood of $x$, denoted $N(x)$, is the set

$$
N(x)=\{y \in \mathcal{X} \mid y \text { is a neighbor of } x\} .
$$

Observe that if $E$ is any edge of $\mathcal{H}$ and $x \in E$, then $E \subseteq N(x) \cup\{x\}$.

Definition 5.5 A $d$-uniform properly-connected hypergraph $\mathcal{H}$ is said to be triangulated if for every nonempty subset $\mathcal{Y} \subseteq \mathcal{X}$, the induced subhypergraph $\mathcal{H} \mathcal{Y}$ contains a vertex $x \in \mathcal{Y} \subseteq \mathcal{X}$ such that the induced hypergraph of $\mathcal{H}_{\mathcal{Y}}$ on $N(x) \cup\{x\}$ is a $d$-complete hypergraph of order $|N(x)|+1$.

By virtue of Theorem 5.2, the simple graphs that are triangulated are precisely the chordal graphs. We shall show that properly-connected hyperforests are triangulated hypergraphs.

Theorem 5.6 Suppose that $\mathcal{H}$ is a d-uniform properly-connected hypergraph that is a $v$-forest (or equivalently, $f$-forest). Then $\mathcal{H}$ is a triangulated hypergraph.

Proof For any $\mathcal{Y} \subseteq \mathcal{X}$, the induced subgraph $\mathcal{H} \mathcal{Y}$ must contain a $v$-leaf, say $E$. Since $E$ is a $v$-leaf, $E$ contains a free vertex, say $x$. Suppose that $E=\left\{x, x_{2}, \ldots, x_{d}\right\}$. Then $N(x)=\left\{x_{2}, \ldots, x_{d}\right\}$. But the induced graph of $\mathcal{H}_{\mathcal{Y}}$ on $N(x) \cup\{x\}$ is simply the edge $E$ which is the $d$-uniform complete hypergraph $\mathcal{K}_{d}^{d}$. So $\mathcal{H}$ is a triangulated hypergraph.

The following lemma is the key result needed to prove that Theorem 4.16 is recursive for triangulated hypergraphs.

Lemma 5.7 Let $\mathcal{H}$ be a triangulated hypergraph. Then there exists an edge $E \in \mathcal{H}$ such that

(a) $E$ is a splitting edge, and

(b) the subgraphs $\mathcal{H} \backslash E$ and $\mathcal{H}^{\prime}$ are triangulated hypergraphs.

Proof Since $\mathcal{H}$ is a triangulated hypergraph, there exists a vertex $x \in \mathcal{X}$ such that the induced hypergraph on $N(x) \cup\{x\}$ is a $d$-complete hypergraph. Let $E$ be any edge of $\mathcal{H}$ that contains $x$. We will show that $E$ is an edge that satisfies $(a)$ and $(b)$. 
(a) Suppose that $N(E)=\left\{z_{1}, \ldots, z_{t}\right\}$. For each $z_{i} \in N(E)$, there must be an edge $E_{i} \in \mathcal{H}$ such that $\operatorname{dist}_{\mathcal{H}}\left(E, E_{i}\right)=1$ and $E \cup E_{i}=E \cup\left\{z_{i}\right\}$. For each $i$, either $x \in E_{i}$ or $x \notin E_{i}$. If $x \notin E_{i}$, then $(E \backslash\{x\}) \cup\left\{z_{i}\right\}=E_{i} \in \mathcal{H}$. Now, suppose that $x \in E_{i}$. Since $z_{i} \in E_{i}$, we have $z_{i} \in N(x)$. If $E=\left\{x, x_{2}, \ldots, x_{d}\right\}$, then $\left\{x_{2}, \ldots, x_{d}, z_{i}\right\} \subseteq N(x)$ is a subset of size $d$ in $N(x) \cup\{x\}$. But since the induced hypergraph on $N(x) \cup\{x\}$ is a $d$-complete hypergraph, this means that $\left\{x_{2}, \ldots, x_{d}, z_{i}\right\}$ is an edge of $\mathcal{H}$. This edge is simply $(E \backslash\{x\}) \cup\left\{z_{i}\right\}$. So, $E$ is a splitting edge by Theorem 4.8.

(b) We consider $\mathcal{H} \backslash E$ first. We begin by showing that $\mathcal{H} \backslash E$ is properly-connected. If $H, H^{\prime} \in \mathcal{H} \backslash E$ with $H \cap H^{\prime} \neq \emptyset$, then in $\mathcal{H}$ we also have $H \cap H^{\prime} \neq \emptyset$. Since $\mathcal{H}$ is properly-connected, we can find a proper irredundant chain $H=E_{0}, E_{1}, \ldots, E_{t}=$ $H^{\prime}$, where $t=\operatorname{dist}_{\mathcal{H}}\left(H, H^{\prime}\right)=d-\left|H \cap H^{\prime}\right|$. If $E \notin\left\{E_{1}, \ldots, E_{t-1}\right\}$, then this chain remains a proper irredundant chain in $\mathcal{H} \backslash E$ giving us $t=\operatorname{dist}_{\mathcal{H} \backslash E}\left(H, H^{\prime}\right)=d-$ $\left|H \cap H^{\prime}\right|$.

So suppose that $E \in\left\{E_{1}, \ldots, E_{t-1}\right\}$. Let $x \in E$ be the vertex such that the induced hypergraph on $N(x) \cup\{x\}$ is a $d$-complete hypergraph. Let $E_{i-1}$ and $E_{i+1}$ be the edges that appear immediately before and after $E$, respectively, in the chain $E_{0}, \ldots, E_{t}$. There then exists a vertex $z_{i-1} \in E_{i-1}$ such that $\left\{z_{i-1}\right\}=E_{i-1} \backslash E$ and a vertex $z_{i+1} \in E_{i+1}$ such that $\left\{z_{i+1}\right\}=E_{i+1} \backslash E$. By Lemma 4.9 there are three cases to consider: (i) $x \in E_{i-1}, E$, and $E_{i+1}$, (ii) $x \in E_{i-1}$ and $E$, but $x \notin E_{i+1}$, or (iii) $x \notin E_{i-1}$ but $x \in E$ and $E_{i+1}$. (Lemma 4.9 shows that when moving through the chain, one removes one vertex from an edge and replaces it with another vertex, and furthermore, once you add a vertex to a chain, this vertex appears in all later edges in the chain.) In case (i), let $E^{\prime}=E_{i-1} \cap E_{i+1}$. Note that $\left|E^{\prime}\right|=d-2$. Then $E^{\prime} \cup\left\{z_{i-1}, z_{i+1}\right\}$ is a subset of $N(x) \cup\{x\}$ of size $d$, and since the induced graph on $N(x) \cup\{x\}$ is a $d$-complete hypergraph, this means that $E^{\prime \prime}=E^{\prime} \cup\left\{z_{i-1}, z_{i+1}\right\}$ is an edge of $\mathcal{H}$. The edge $E^{\prime \prime}$ is distance one from $E_{i-1}$ and $E_{i+1}$. We can replace $E$ in the chain $E_{0}, \ldots, E_{t}$ with $E^{\prime \prime}$ and still have a proper chain of length $t$ in $\mathcal{H} \backslash E$ from $H$ to $H^{\prime}$. Moreover, this chain must be irredundant, since if it was shorter, then this would give rise to a shorter chain in $\mathcal{H}$, contradicting the fact that $t$ is the length of the shortest chain. In case $(i i)$, let $z$ be the vertex of $E$ such that $\{z\}=E \backslash E_{i-1}$. Then $E_{i-1} \backslash\{x\}$ and $z$ are in $N(x) \subseteq N(x) \cup\{x\}$. Thus $E^{\prime}=\left(E_{i-1} \backslash\{x\}\right) \cup\{z\}$ also is an edge of $\mathcal{H}$. Furthermore, $E^{\prime}$ is distance one away from $E_{i-1}$ and $E_{i+1}$ (since $z$ is added to $E$, we have $z \in E_{i+1}$ ). So, we can replace $E$ in the chain by $E^{\prime}$ and get a chain of correct length in $\mathcal{H} \backslash E$. Finally, in case (iii), let $z$ be the vertex in $E$ such that $\{z\}=E \backslash E_{i+1}$. Then $z \in N(x)$ and $\left(E_{i+1} \backslash\{x\}\right) \subseteq N(x)$. This means that $E^{\prime}=\left(E_{i+1} \backslash\{x\}\right) \cup\{z\}$ is an edge of $\mathcal{H}$. But this edge is distance one from both $E_{i-1}$ and $E_{i+1}$, so, as we did before, we can replace $E$ with $E^{\prime}$ to get a chain of the desired length.

We can now show that $\mathcal{H} \backslash E$ is also triangulated. If the vertex $x \in E$ only appears in $E$, then $E$ is a $v$-leaf. Then $\mathcal{H} \backslash E=\mathcal{H} \backslash\{x\}=\mathcal{H}_{\mathcal{X} \backslash\{x\}}$, and it is clear that $\mathcal{H}_{\mathcal{X} \backslash\{x\}}$ is a triangulated hypergraph. So, suppose that there are two or more edges that contain $x$. If $\mathcal{Y} \subseteq \mathcal{X}$ with $x \notin \mathcal{Y}$, then the induced hypergraph of $\mathcal{H} \backslash E$ on $\mathcal{Y}$ is the same as the induced hypergraph of $\mathcal{H}$ on $\mathcal{Y}$, so there exists a vertex $z \in \mathcal{Y}$ such that the induced hypergraph on $N(z) \cup\{z\}$ is a $d$-complete hypergraph. It remains to consider the case where $x \in \mathcal{Y}$. Let $N \mathcal{Y}(x)$ denote the neighbors of $x$ in $(\mathcal{H} \backslash E) \mathcal{Y}$. Note that $N \mathcal{Y}(x) \cup\{x\} \subseteq N(x) \cup\{x\}$. Since the induced hypergraph on $N(x) \cup\{x\}$ is a 
$d$-complete hypergraph, any induced subgraph on a subset of $N(x) \cup\{x\}$ also is a $d$-complete hypergraph. So the induced hypergraph $(\mathcal{H} \backslash E)_{N_{\mathcal{Y}}(x) \cup\{x\}}$ is a $d$-complete hypergraph. Thus $\mathcal{H} \backslash E$ is triangulated.

Finally, by Lemma 4.13 we know that $\mathcal{H}^{\prime}$ is properly-connected. The reason that $\mathcal{H}^{\prime}$ is triangulated follows from the fact that

$$
\mathcal{H}^{\prime}=\mathcal{H} \backslash\left\{x, x_{2}, \ldots, x_{d}, z_{1}, \ldots, z_{t}\right\}=\mathcal{H}_{\mathcal{X} \backslash\left\{x, x_{2}, \ldots, x_{d}, z_{1}, \ldots, z_{t}\right\}},
$$

where $E=\left\{x, x_{2}, \ldots, x_{d}\right\}$ and $N(E)=\left\{z_{1}, \ldots, z_{t}\right\}$.

We come to the main result of this section.

Theorem 5.8 Suppose that $\mathcal{H}$ is a d-uniform triangulated hypergraph. Then the graded Betti numbers of $\mathcal{I}(\mathcal{H})$ can be computed recursively using the formula

$$
\beta_{i, j}(\mathcal{I}(\mathcal{H}))=\beta_{i, j}(\mathcal{I}(\mathcal{H} \backslash E))+\sum_{l=0}^{i}\left(\begin{array}{l}
t \\
l
\end{array}\right) \beta_{i-1-l, j-d-l}\left(\mathcal{I}\left(\mathcal{H}^{\prime}\right)\right),
$$

where $E$ is a splitting edge, $t=|N(E)|$, and $\mathcal{H}^{\prime}$ and $\mathcal{H} \backslash E$ are also d-uniform triangulated hypergraphs. Here, $\beta_{-1, j}\left(\mathcal{I}\left(\mathcal{H}^{\prime}\right)\right)=1$ if $j=0$ and 0 if $j \neq 0$.

Proof By Lemma 5.7, the triangulated hypergraph $\mathcal{H}$ has a splitting edge $E$. Furthermore, since both hypergraphs $\mathcal{H} \backslash E$ and $\mathcal{H}^{\prime}$ are triangulated hypergraphs, they also have splitting edges. Thus, by repeatedly using the formula of Theorem 4.16 we get the recursive formula.

It is well known that the graded Betti numbers for an arbitrary monomial ideal may depend upon the characteristic of $k$. However, as a consequence of the above formula, we obtain the following corollary.

Corollary 5.9 Suppose that $\mathcal{H}$ is a triangulated hypergraph. Then the graded Betti numbers of $\mathcal{I}(\mathcal{H})$ are independent of the characteristic of the ground field and can be computed recursively.

When restricted to simple graphs, we get a particularly nice corollary.

Corollary 5.10 Suppose that $G$ is a chordal graph. Then the graded Betti numbers of $\mathcal{I}(G)$ are independent of the characteristic of the ground field and can be computed recursively.

Jacques [19] and Jacques and Katzman [20] first proved Corollary 5.10 in the special case that $G$ is a forest, a subclass of chordal graphs.

\section{Properly-connected hypergraphs and regularity}

In this section we investigate the Castelnuovo-Mumford regularity of the edge ideal $\mathcal{I}(\mathcal{H})$ associated to a properly-connected hypergraph $\mathcal{H}$. For such a hypergraph, we 
bound $\operatorname{reg}(\mathcal{I}(\mathcal{H}))$ below by combinatorial invariants of the hypergraph. When $\mathcal{H}=G$ is a simple graph, we also provide an upper bound. In the case that $\mathcal{H}$ is also triangulated, we explicitly compute $\operatorname{reg}(\mathcal{I}(\mathcal{H}))$. Our exact formula for $\operatorname{reg}(\mathcal{I}(\mathcal{H}))$ generalizes Zheng's formula [33] for the regularity of $\mathcal{I}(\mathcal{H})$ when $\mathcal{H}=G$ is a forest.

We begin by relating the regularity of $\mathcal{I}(\mathcal{H})$ to the regularity of edge ideals associated to sub-hypergraphs of $\mathcal{H}$. We produce similar results for the projective dimension of $\mathcal{I}(\mathcal{H})$. We first make the convention that $\operatorname{reg}(0)=1$ and if $\mathcal{H}$ has no edges, we set $\operatorname{pdim}(\mathcal{I}(\mathcal{H}))=-1$.

Lemma 6.1 Let $E$ be any edge of a $d$-uniform properly-connected hypergraph $\mathcal{H}$ such that $\mathcal{H} \backslash E$ is nonempty. Let $t=|N(E)|$ and $\mathcal{H}^{\prime}=\left\{H \in \mathcal{H} \mid \operatorname{dist}_{\mathcal{H}}(H, E) \geq d+\right.$ 1\}. If $L=\left(x^{E}\right) \cap \mathcal{I}(\mathcal{H} \backslash E)$, then

(a) $\operatorname{reg}(L)=\operatorname{reg}\left(\mathcal{I}\left(\mathcal{H}^{\prime}\right)\right)+d$, and

(b) $\operatorname{pdim}(L)=\operatorname{pdim}\left(\mathcal{I}\left(\mathcal{H}^{\prime}\right)\right)+t$.

Proof We shall prove both results using Lemma 4.15. For $(a)$, suppose that $s=$ $\operatorname{reg}(L)$. So, there exists $a$ such that $\beta_{a, a+s}(L) \neq 0$. By Lemma 4.15,

$$
\beta_{a+1-1, a+s}(L)=\sum_{l=0}^{a+1}\left(\begin{array}{l}
t \\
l
\end{array}\right) \beta_{a+1-1-l, a+s-d-l}\left(\mathcal{I}\left(\mathcal{H}^{\prime}\right)\right)
$$

Since every number in the summation on the right-hand side is nonnegative, there exists $l$ such that $\beta_{a-l, a+s-d-l}\left(\mathcal{I}\left(\mathcal{H}^{\prime}\right)\right) \neq 0$. Hence, $\operatorname{reg}\left(\mathcal{I}\left(\mathcal{H}^{\prime}\right)\right) \geq s-d$, or equivalently, $\operatorname{reg}\left(\mathcal{I}\left(\mathcal{H}^{\prime}\right)\right)+d \geq \operatorname{reg}(L)$. Conversely, if $r=\operatorname{reg}\left(\mathcal{I}\left(\mathcal{H}^{\prime}\right)\right)$, then there exists $b$ such that $\beta_{b, b+r}\left(\mathcal{I}\left(\mathcal{H}^{\prime}\right)\right) \neq 0$. But then, since $b+r=(b+r+d)-d$, by Lemma 4.15 we have

$$
0 \neq \beta_{b,(b+r+d)-d}\left(\mathcal{I}\left(\mathcal{H}^{\prime}\right)\right) \leq \sum_{l=0}^{b+1}\left(\begin{array}{l}
t \\
l
\end{array}\right) \beta_{b+1-1-l, b+r+d-d-l}\left(\mathcal{I}\left(\mathcal{H}^{\prime}\right)\right)=\beta_{b, b+r+d}(L)
$$

So $\operatorname{reg}\left(\mathcal{I}\left(\mathcal{H}^{\prime}\right)\right)+d \geq \operatorname{reg}(L) \geq \operatorname{reg}\left(\mathcal{I}\left(\mathcal{H}^{\prime}\right)\right)+d$, as desired.

To prove $(b)$, suppose that $N(E)=\left\{z_{1}, \ldots, z_{t}\right\}$. In the proof of Lemma 4.15 it was shown that

$$
R / L \cong R_{1} /\left(z_{1}, \ldots, z_{t}\right) \otimes_{k} R_{2} / \mathcal{I}\left(\mathcal{H}^{\prime}\right),
$$

where $R_{1}=k\left[z_{1}, \ldots, z_{t}\right]$ and $R_{2}=k\left[x_{1}, \ldots, x_{s}\right]$ with $\left\{x_{1}, \ldots, x_{s}\right\}=\mathcal{X} \backslash N(E)$. By tensoring the resolutions of $R_{1} /\left(z_{1}, \ldots, z_{t}\right)$ and $R_{2} / \mathcal{I}\left(\mathcal{H}^{\prime}\right)$, we get

$$
\begin{aligned}
\operatorname{pdim}(L)+1 & =\operatorname{pdim}(R / L)=\operatorname{pdim}\left(R_{1} /\left(z_{1}, \ldots, z_{t}\right)\right)+\operatorname{pdim}\left(R_{2} / \mathcal{I}\left(\mathcal{H}^{\prime}\right)\right) \\
& =t+\operatorname{pdim}\left(R / \mathcal{I}\left(\mathcal{H}^{\prime}\right)\right)=t+\operatorname{pdim}\left(\mathcal{I}\left(\mathcal{H}^{\prime}\right)\right)+1
\end{aligned}
$$

The desired identity is obtained by comparing the first and last values of the above equality.

Theorem 6.2 Let $E$ be any edge of a d-uniform properly-connected hypergraph $\mathcal{H}$ such that $\mathcal{H} \backslash E$ is nonempty. Let $t=|N(E)|$. Then 
(a) $\operatorname{reg}(\mathcal{I}(\mathcal{H})) \leq \max \left\{\operatorname{reg}(\mathcal{I}(\mathcal{H} \backslash E)), \operatorname{reg}\left(\mathcal{I}\left(\mathcal{H}^{\prime}\right)\right)+d-1\right\}$;

(b) $\operatorname{pdim}(\mathcal{I}(\mathcal{H})) \leq \max \left\{\operatorname{pdim}(\mathcal{I}(\mathcal{H} \backslash E)), \operatorname{pdim}\left(\mathcal{I}\left(\mathcal{H}^{\prime}\right)\right)+t+1\right\}$.

Furthermore, if $E$ is a splitting edge, then we have equality in both (a) and (b).

Proof Set $L=\left(x^{E}\right) \cap \mathcal{I}(\mathcal{H} \backslash E)$. The two inequalities then follow by using the short exact sequence

$$
0 \rightarrow L \rightarrow\left(x^{E}\right) \oplus \mathcal{I}(\mathcal{H} \backslash E) \rightarrow \mathcal{I}(\mathcal{H}) \rightarrow 0
$$

and Lemma 6.1 to bound $\operatorname{reg}(I(\mathcal{H}))$ and $\operatorname{pdim}(\mathcal{I}(\mathcal{H}))$, noting that since $\mathcal{H} \backslash E$ is nonempty, $\operatorname{reg}(\mathcal{H} \backslash E) \geq d$. When $E$ is a splitting edge, the equalities are a result of the formulas of Corollary 2.5.

We now focus our attention on using combinatorial information from $\mathcal{H}$ to bound $\operatorname{reg}(\mathcal{I}(\mathcal{H}))$. More precisely, the regularity will be expressed using the following terminology.

Definition 6.3 Let $\mathcal{H}$ be a $d$-uniform properly-connected hypergraph. Two edges $E, H$ of $\mathcal{H}$ are $t$-disjoint if $\operatorname{dist}_{\mathcal{H}}(E, H) \geq t$. A set of edges $\mathcal{E}^{\prime} \subseteq \mathcal{E}$ is pairwise $t$ disjoint if every pair of edges of $\mathcal{E}^{\prime}$ is $t$-disjoint. (We thank Jeremy Martin for suggesting this name.)

Remark 6.4 When $\mathcal{H}$ is a $d$-uniform properly-connected hypergraph, then two edges $E$ and $H$ are $d$-disjoint if and only if $E \cap H=\emptyset$; that is, $E$ and $H$ are disjoint in the usual sense. When $\mathcal{H}=G$ is a simple graph, Zheng's definition [33, Definition 2.15] for two edges to be disconnected is equivalent to our definition that the two edges be 3-disjoint in $G$.

We come to the first main result of this section.

Theorem 6.5 Let $\mathcal{H}$ be a d-uniform properly-connected hypergraph. Then $\beta_{i-1, d i}(\mathcal{I}(\mathcal{H}))$ equals the number of sets of $i$ pairwise $(d+1)$-disjoint edges of $\mathcal{H}$. In particular, if $c$ is the maximal number of pairwise $(d+1)$-disjoint edges of $\mathcal{H}$, then

$$
\operatorname{reg}(\mathcal{I}(\mathcal{H})) \geq(d-1) c+1
$$

Proof The first statement of the theorem implies that $\beta_{c-1, d c}(\mathcal{I}(\mathcal{H})) \neq 0$. Thus, $d c-$ $(c-1) \leq \operatorname{reg}(\mathcal{I}(\mathcal{H}))$, and the second statement is proved. We shall prove the first statement of the theorem. In the case $d=2$, this is the content of [21, Lemma 2.2]. We generalize Katzman's arguments to the more general situation.

Recall that $\mathcal{E}=\left\{E_{1}, \ldots, E_{s}\right\}$ and let $\mathbb{T}: 0 \rightarrow T_{s} \stackrel{\partial_{s}}{\rightarrow} \cdots \stackrel{\partial_{2}}{\rightarrow} T_{1} \stackrel{\partial_{1}}{\rightarrow} \mathcal{I}(\mathcal{H}) \rightarrow 0$ be the Taylor resolution of $\mathcal{I}(\mathcal{H})$. Then $T_{i}$ is a free $R$-module with generators $e_{j_{1}, \ldots, j_{i}}$, for $1 \leq j_{1}<\cdots<j_{i} \leq s$, and the boundary map $\partial_{i}$ is defined by

$$
\partial_{i}\left(e_{j_{1}, \ldots, j_{i}}\right)=\sum_{k=1}^{i}(-1)^{k} \mu_{k} e_{j_{1}, \ldots, \hat{j}_{k}, \ldots, j_{i}},
$$


where $\widehat{j_{k}}$ indicates the removal of $j_{k}$, and $\mu_{k}=x^{E_{j_{k}} \backslash\left(\cup_{l \neq k} E_{j_{l}}\right)}$. Let $\mathfrak{m}=\left(x_{1}, \ldots, x_{n}\right)$ be the maximal homogeneous ideal in $R$. It is well known that the graded Betti numbers of $\mathcal{I}(\mathcal{H})$ are given by

$$
\beta_{i-1, j}(\mathcal{I}(\mathcal{H}))=\operatorname{dim}_{k} H_{i}\left(\mathbb{T} \otimes_{R} R / \mathfrak{m}\right)_{j}
$$

Observe that generators of degree $d i$ of $T_{i}$ are $e_{j_{1}, \ldots, j_{i}}$ 's, where $E_{j_{1}}, \ldots, E_{j_{i}}$ are pairwise disjoint. Consider one such generator $e_{j_{1}, \ldots, j_{i}}$. Let $\mathcal{H}_{1}$ be the induced subhypergraph of $\mathcal{H}$ on the vertices in $\bigcup_{k=1}^{i} E_{j_{k}}$. It can be seen that for $1 \leq k \leq i$, $E_{j_{k}}$ is disjoint from $\bigcup_{l \neq k} E_{j_{l}}$ and hence, $\mu_{k} \in \mathfrak{m}$. Thus, the image of $\partial_{i}\left(e_{j_{1}, \ldots, j_{i}}\right)$ in $\mathbb{T} \otimes_{R} R / \mathfrak{m}$ is 0 . Also, if $\mathcal{H}_{1}$ contains an edge $E_{t}$ different from $E_{j_{1}}, \ldots, E_{j_{i}}$, then since $E_{t} \subseteq \bigcup_{k=1}^{i} E_{j_{k}}$, we have $\partial_{i+1}\left(e_{j_{1}, \ldots, j_{i}, t}\right)=e_{j_{1}, \ldots, j_{i}}$. That is, if $\mathcal{H}_{1}$ contains an edge different from $E_{j_{1}}, \ldots, E_{j_{i}}$, then the image of $e_{j_{1}, \ldots, j_{i}}$ in $H_{i}\left(\mathbb{T} \otimes_{R} R / \mathfrak{m}\right)$ is 0 . Furthermore, the image of $e_{j_{1}, \ldots, j_{i}}$ in $\mathbb{T} \otimes_{R} R / \mathfrak{m}$ is in the image of $\partial_{i+1}$ if and only if it is the image of $\partial_{i+1}\left(e_{l_{1}, \ldots, l_{i+1}}\right)$, where $\left\{l_{1}, \ldots, l_{i+1}\right\}=\left\{j_{1}, \ldots, j_{i}\right\} \cup\{t\}$ for some $t$. This implies that in the expansion of $\partial_{i+1}\left(e_{l_{1}, \ldots, l_{i+1}}\right)$, we must have $\mu_{t}=1$, i.e., $\mathcal{H}_{1}$ contains the edge $E_{t}$ different from $E_{j_{1}}, \ldots, E_{j_{i}}$.

It remains to show that $E_{j_{1}}, \ldots, E_{j_{i}}$ are pairwise disjoint edges of $\mathcal{H}$ such that the induced sub-hypergraph of $\mathcal{H}$ on the vertices of $\bigcup_{k=1}^{i} E_{j_{k}}$ contains no other edges if and only if $E_{j_{1}}, \ldots, E_{j_{i}}$ are pairwise $(d+1)$-disjoint edges in $\mathcal{H}$.

Suppose first that $E_{j_{1}}, \ldots, E_{j_{i}}$ are pairwise disjoint edges of $\mathcal{H}$ such that the induced sub-hypergraph $\mathcal{H}_{1}$ on the vertices of $\bigcup_{k=1}^{i} E_{j_{k}}$ contains no other edges. Clearly, since $E_{j_{k}} \cap E_{j_{l}}=\emptyset$ for $k \neq l$, we have $\operatorname{dist}_{\mathcal{H}}\left(E_{j_{k}}, E_{j_{l}}\right) \geq d$. Now, suppose that there exist $k \neq l$ such that $\operatorname{dist}_{\mathcal{H}}\left(E_{j_{k}}, E_{j_{l}}\right)=d$. Then there is a proper chain $E_{j_{k}}=F_{0}, F_{1}, \ldots, F_{d}=E_{j_{l}}$. By Lemma 4.9, the vertices of $F_{1}$ are in $E_{j_{k}} \cup E_{j_{l}}$, so $F_{1}$ is an edge in $\mathcal{H}_{1}$. This implies that $F_{1}$ has to be one of the $\left\{E_{j_{1}}, \ldots, E_{j_{i}}\right\} \backslash\left\{E_{j_{k}}, E_{j_{l}}\right\}$. This is a contradiction since $F_{1} \cap E_{j_{k}} \neq \emptyset$.

Conversely, suppose that $E_{j_{1}}, \ldots, E_{j_{i}}$ are pairwise $(d+1)$-disjoint edges of $\mathcal{H}$. Let $\mathcal{H}_{1}$ be the induced sub-hypergraph of $\mathcal{H}$ on the vertices of $\bigcup_{k=1}^{i} E_{j_{k}}$. By contradiction, suppose that $\mathcal{H}_{1}$ contains an edge $E$ different from $E_{j_{1}}, \ldots, E_{j_{i}}$. Then $E \subseteq \bigcup_{k=1}^{i} E_{j_{k}}$. Without loss of generality, we may assume that $E \cap E_{j_{1}} \neq \emptyset$. Then there is a proper chain $E_{j_{1}}=F_{0}, F_{1}, \ldots, F_{l}=E$ for some $l<d$. By Lemma 4.9, the vertices of $F_{1}$ are in $E_{j_{1}} \cup E$. Thus, $F_{1}$ also is an edge of $\mathcal{H}_{1}$. This implies that there exists $j_{k} \neq j_{1}$ such that $F_{1}$ has a nonempty intersection with $E_{j_{k}}$ (otherwise, $F_{1} \subseteq E_{j_{1}}$, which is a contradiction). However, we now have $\operatorname{dist}_{\mathcal{H}}\left(F_{1}, E_{j_{k}}\right)=$ $d-\left|F_{1} \cap E_{j_{k}}\right| \leq d-1$, whence $\operatorname{dist}_{\mathcal{H}}\left(E_{j_{1}}, E_{j_{k}}\right) \leq d$, which is again a contradiction.

When $\mathcal{H}$ is a graph, we also obtain an especially appealing upper bound for the regularity of $\mathcal{I}(\mathcal{H})$.

Definition 6.6 Let $\mathcal{H}=(\mathcal{X}, \mathcal{E})$ be a hypergraph. A matching of $\mathcal{H}$ is defined to be a subset $\mathcal{E}^{\prime} \subseteq \mathcal{E}$ consisting of pairwise disjoint edges. The matching number of $\mathcal{H}$, denoted $\alpha^{\prime}(\mathcal{H})$, is the largest size of a maximal matching in $\mathcal{H}$. 
Theorem 6.7 Let $G$ be a finite simple graph. Then

$$
\operatorname{reg}(R / \mathcal{I}(G)) \leq \alpha^{\prime}(G),
$$

where $\alpha^{\prime}(G)$ is the matching number of $G$.

Proof It can be seen from the Taylor resolution that

$$
\operatorname{reg}(\mathcal{I}(G)) \leq \max \left\{\operatorname{deg} \operatorname{lcm}\left(x^{E_{1}}, \ldots, x^{E_{i}}\right)-i \mid\left\{E_{1}, \ldots, E_{i}\right\} \subseteq \mathcal{E}\right\}+1 .
$$

Since any edge of $G$ has 2 vertices, it can be seen that $i+1 \leq \operatorname{deg} \operatorname{lcm}\left(x^{E_{1}}, \ldots, x^{E_{i}}\right) \leq$ $2 i$. Let $\operatorname{deg} \operatorname{lcm}\left(x^{E_{1}}, \ldots, x^{E_{i}}\right)=i+k$ for some $1 \leq k \leq i$. It suffices to show that we can always find a matching of size $k$ among $\left\{E_{1}, \ldots, E_{i}\right\}$. To this end, we shall use induction on $i+k$.

If $i+k=2$, i.e., $i=k=1$, then the statement is clear. Suppose now that $i+k>2$. If $k=1$ or $k=i$, then the statement is also clear. Assume that $1<k<i$. If $E_{i}$ is disjoint from $E_{j}$ for all $j<i$, then $\operatorname{deg} \operatorname{lcm}\left(x^{E_{1}}, \ldots, x^{E_{i-1}}\right)=i+k-2=(i-1)+$ $(k-1)$. By induction, there exists a matching $S \subset\left\{E_{1}, \ldots, E_{i-1}\right\}$ of size $(k-1)$. It is easy to see that $S \cup\left\{E_{i}\right\}$ is now a matching of size $k$. It remains to consider the case that at least a vertex of $E_{i}$ is also a vertex of $E_{j}$ for some $j<i$. In this case, we have $\operatorname{deg} \operatorname{lcm}\left(x^{E_{1}}, \ldots, x^{E_{i-1}}\right) \geq i+k-1=(i-1)+k$. By induction, there is a matching $S \subset\left\{E_{1}, \ldots, E_{i-1}\right\}$ of size $k$, and the statement is proved.

Theorem 6.7 seems to give an interesting bound for the regularity of edge ideals with a simple proof which may have been overlooked.

When $\mathcal{H}$ is a triangulated hypergraph, the lower bound of Theorem 6.5 turns out to be the exact formula for the regularity of $\mathcal{I}(\mathcal{H})$.

Theorem 6.8 Suppose that $\mathcal{H}$ is a d-uniform properly-connected triangulated hypergraph. If $c$ is the maximum number of pairwise $(d+1)$-disjoint edges of $\mathcal{H}$, then

$$
\operatorname{reg}(\mathcal{I}(\mathcal{H}))=(d-1) c+1
$$

Proof The proof is similar to the one given by [22] in the case for forests. We proceed by induction on the number of edges of $\mathcal{H}$. If $\mathcal{H}$ only has one edge $E$, then $\mathcal{I}(\mathcal{H})=$ $\left(x^{E}\right)$. Since $\mathcal{I}(\mathcal{H})$ is principal, it is clear that $\operatorname{reg}(\mathcal{I}(\mathcal{H}))=d$. But then it is clear that the formula holds since 1 is the maximal number of pairwise $(d+1)$-disjoint edges.

So, suppose that $\mathcal{H}$ has at least two edges. Since $\mathcal{H}$ is triangulated, by Lemma 5.7 there is a splitting edge $E \in \mathcal{H}(\mathcal{H} \backslash E$ is nonempty in this case) such that $\mathcal{H} \backslash E$ and $\mathcal{H}^{\prime}$ are also $d$-uniform properly-connected triangulated hypergraphs. Since $E$ is a splitting edge, by Corollary 6.2 we have

$$
\operatorname{reg}(\mathcal{I}(\mathcal{H}))=\max \left\{\operatorname{reg}(\mathcal{I}(\mathcal{H} \backslash E)), \operatorname{reg}\left(\mathcal{I}\left(\mathcal{H}^{\prime}\right)\right)+d-1\right\}
$$

By induction $\operatorname{reg}(\mathcal{I}(\mathcal{H} \backslash E))=(d-1) c_{1}+1$, where $c_{1}$ is the maximal number of pairwise $(d+1)$-disjoint edges of $\mathcal{H} \backslash E$, and $\operatorname{reg}\left(\mathcal{I}\left(\mathcal{H}^{\prime}\right)\right)=(d-1) c_{2}+1$, where $c_{2}$ is the maximal number of pairwise $(d+1)$-disjoint edges of $\mathcal{H}^{\prime}$. So

$$
\operatorname{reg}(\mathcal{I}(\mathcal{H}))=\max \left\{(d-1) c_{1}+1,(d-1) c_{2}+d\right\}
$$


If we let $c$ denote the maximal number of pairwise $(d+1)$-disjoint edges of $\mathcal{H}$, then since $(d-1) c_{2}+d=(d-1)\left(c_{2}+1\right)+1$, to complete the proof it suffices for us to show that $c=\max \left\{c_{1}, c_{2}+1\right\}$.

Let $\mathcal{E}_{1}$ be the set of the $c_{1}$ pairwise $(d+1)$-disjoint edges of $\mathcal{H} \backslash E$. The edges of $\mathcal{E}_{1}$ are also a set of pairwise $d+1$-disjoint edges of $\mathcal{H}$. To see this fact, suppose that $H, H^{\prime}$ are two $(d+1)$-disjoint edges in $\mathcal{H} \backslash E$ that are not $(d+1)$-disjoint in $\mathcal{H}$. That is, $\operatorname{dist}_{\mathcal{H}}\left(H, H^{\prime}\right) \leq d$. But since $H \cap H^{\prime}=\emptyset$, we must have $\operatorname{dist}_{\mathcal{H}}\left(H, H^{\prime}\right)=d$. Let $H=E_{0}, \ldots, E_{d}=H^{\prime}$ be the proper irredundant chain of length $d$ in $\mathcal{H}$. Since this chain is not in $\mathcal{H} \backslash E$, we must have $E=E_{i}$ for some $i=\{1, \ldots, d-1\}$. Consider the edges $E_{i-1}$ and $E_{i+1}$ in the chain that occur before and after, respectively, the edge $E$. The splitting edge $E$ of Lemma 5.7 is picked so that it contains a vertex $x$ such that the induced graph on $N(x) \cup\{x\}$ is a $d$-complete hypergraph. We can now adapt the proof given in Lemma 5.7 that showed that $\mathcal{H} \backslash E$ was properly-connected to show that $E$ can be replaced by an edge $E^{\prime} \in \mathcal{H} \backslash E$. As a consequence, we get a path of length $d$ from $H$ to $H^{\prime}$ in $\mathcal{H} \backslash E$. But this contradicts the fact that $\operatorname{dist}_{\mathcal{H} \backslash E}\left(H, H^{\prime}\right) \geq d+1$. Thus $\left|\mathcal{E}_{1}\right|=c_{1} \leq c$.

If $\mathcal{E}_{2}$ is a set of $c_{2}$ pairwise $(d+1)$-disjoint edges of $\mathcal{H}^{\prime}$, we claim that $\mathcal{E}_{2} \cup$ $\{E\}$ is a set of pairwise $(d+1)$-disjoint edges of $\mathcal{H}$. Indeed, for any edge $H \in \mathcal{H}^{\prime}$, $\operatorname{dist}_{\mathcal{H}}(E, H)>d$, and so in particular, $E$ and $H$ are $(d+1)$-disjoint for every edge $H \in \mathcal{E}_{2}$. Thus $\left|\mathcal{E}_{2} \cup\{E\}\right|=c_{2}+1 \leq c$. Thus $c \geq \max \left\{c_{1}, c_{2}+1\right\}$.

Suppose that $c>\max \left\{c_{1}, c_{2}+1\right\}$. Let $\mathcal{E}$ be a set of $c$ pairwise $(d+1)$-disjoint edges of $\mathcal{H}$. If $E \notin \mathcal{E}$, then $\mathcal{E}$ also is a set of pairwise $(d+1)$-disjoint edges of $\mathcal{H} \backslash E$, and so $c=|\mathcal{E}| \leq c_{1}$, a contradiction. If $E \in \mathcal{E}$, then $\mathcal{E} \backslash\{E\}$ is a set of pairwise $(d+1)$ disjoint edges of $\mathcal{H}^{\prime}$, since any other edge $H \in \mathcal{E}$ must have $\operatorname{dist}_{\mathcal{H}}(E, H)>d$. But this would imply that $c-1 \leq c_{2}$, again a contradiction. Hence $c=\max \left\{c_{1}, c_{2}+1\right\}$.

Theorem 6.8 gives the following interesting corollary for simple graphs, which was first proved by Zheng [33] in the special case that $G$ was a forest.

Corollary 6.9 Suppose that $G$ is chordal graph. If $c$ is the maximum number of pairwise 3-disjoint edges of $G$, then

$$
\operatorname{reg}(\mathcal{I}(G))=c+1
$$

Example 6.10 The bounds for the regularity in Theorems 6.5 and Theorem 6.7 are sharp. If $\mathcal{H}$ is any triangulated hypergraph, then the lower bound in Theorem 6.5 is achieved by Theorem 6.8. To show that the upper bound in Theorem 6.7 is achieved, consider the edge ideal of $C_{5}$, the five-cycle. So $\mathcal{I}(G)=$ $\left(x_{1} x_{2}, x_{2} x_{3}, x_{3} x_{4}, x_{4} x_{5}, x_{5} x_{1}\right)$. Then $\alpha^{\prime}(G)=2$ (for example, take edges $E_{1}=x_{1} x_{2}$ and $\left.E_{2}=x_{3} x_{4}\right)$. So $\operatorname{reg}(\mathcal{I}(G)) \leq 3$. In fact we have equality, since the resolution of $\mathcal{I}(G)$ is

$$
0 \rightarrow R(-5) \rightarrow R^{5}(-3) \rightarrow R^{5}(-2) \rightarrow \mathcal{I}(G) \rightarrow 0
$$

In the study of squarefree monomial ideals, the theory of Alexander duality has proved to be significant in many ways. We round out this section by relating some algebraic invariants of edge ideals and their Alexander duals. 
Definition 6.11 Let $I=\left(x_{11} \cdots x_{1 i_{1}}, \ldots, x_{r 1} \cdots x_{r i_{r}}\right) \subseteq k\left[x_{1}, \ldots, x_{n}\right]$ be a squarefree monomial ideal. Then the Alexander dual of $I$ is defined to be

$$
I^{\vee}=\left(x_{11}, \ldots, x_{1 i_{1}}\right) \cap \cdots \cap\left(x_{r 1}, \ldots, x_{r i_{r}}\right) .
$$

Definition 6.12 Let $G$ be a graph. A subset $V$ of the vertices of $G$ is called a vertex cover if every edge in $G$ is incident to at least a vertex in $V$; a minimal vertex cover is a vertex cover $V$ with the property that no proper subset of $V$ is. The smallest size of a minimal vertex cover of $G$ is denoted by $v(G)$. The graph $G$ is unmixed if all its minimal vertex covers have the same cardinality $v(G)$.

Remark 6.13 The operation of taking the Alexander dual of a squarefree monomial ideal brings generators to primary components. The minimal generators of $\mathcal{I}(G)^{\vee}$ correspond to minimal vertex covers of $G$.

Theorem 6.14 Let $G$ be a simple graph.

(1) If $G$ is unmixed, then

$$
\begin{aligned}
& \operatorname{reg}(\mathcal{I}(G)) \leq \operatorname{ht} \mathcal{I}(G)+1 \leq \operatorname{reg}\left(\mathcal{I}(G)^{\vee}\right)+1 \quad \text { and } \\
& \operatorname{pdim}\left(\mathcal{I}(G)^{\vee}\right) \leq \operatorname{ht} \mathcal{I}(G) \leq \operatorname{pdim}(\mathcal{I}(G))+1 .
\end{aligned}
$$

(2) If $G$ is not unmixed, then

$$
\begin{aligned}
& \operatorname{reg}(\mathcal{I}(G)) \leq \operatorname{ht} \mathcal{I}(G)+1 \leq \operatorname{reg}\left(\mathcal{I}(G)^{\vee}\right) \quad \text { and } \\
& \operatorname{pdim}\left(\mathcal{I}(G)^{\vee}\right) \leq \operatorname{ht} \mathcal{I}(G) \leq \operatorname{pdim}(\mathcal{I}(G)) .
\end{aligned}
$$

Proof It suffices to prove the inequalities involving the regularity, since the bounds on the projective dimension follow from the identities $\operatorname{reg}(\mathcal{I}(G))=\operatorname{pdim}\left(R / \mathcal{I}(G)^{\vee}\right)$ and $\operatorname{reg}\left(\mathcal{I}(G)^{\vee}\right)=\operatorname{pdim}(R / \mathcal{I}(G)$ ) (see, for example, [24, Theorem 5.59]). Observe that if $\mathcal{E}^{\prime}$ is a matching in $G$, then any vertex cover must contain at least a vertex of every edge in $\mathcal{E}^{\prime}$. Thus, $\alpha^{\prime}(G) \leq v(G)=$ ht $\mathcal{I}(G)$. From Theorem 6.7 it follows that $\operatorname{reg}(\mathcal{I}(G)) \leq \operatorname{ht} \mathcal{I}(G)+1$. Since $v(G)$ is the least generating degree of $\mathcal{I}(G)^{\vee}$, we have $v(G) \leq \operatorname{reg}\left(\mathcal{I}(G)^{\vee}\right)$, and thus (1) follows. To prove (2) observe that when $G$ is not unmixed, $\operatorname{reg}\left(\mathcal{I}(G)^{\vee}\right)$ is at least the largest generating degree of $\mathcal{I}(G)^{\vee}$, which is at least $v(G)+1$.

\section{Properly-connected hypergraphs and linear first syzygies}

In [14] Fröberg gave a characterization of edge ideals of simple graphs with linear resolutions. In this section, we obtain a partial generalization of Fröberg's result to the class of properly-connected hypergraphs. Specifically, we describe when $\mathcal{I}(\mathcal{H})$ has linear first syzygies.

Let us first recall Fröberg's result. If $G$ is a simple graph, then the complement of $G$, denoted $G^{c}$, is the graph whose vertex set is the same as that of $G$, but whose edge set is defined by the rule $E \in G^{c}$ if and only $E \notin G$. Fröberg then showed: 
Theorem 7.1 Let $G$ be a simple graph. Then $\mathcal{I}(G)$ has a linear resolution if and only if $G^{c}$ is a chordal graph.

When $\mathcal{H}$ is a $d$-uniform properly-connected hypergraph, we define the complement of $\mathcal{H}$, denoted $\mathcal{H}^{c}$, as

$$
\mathcal{H}^{c}=\{E \subseteq \mathcal{X}|| E \mid=d \text { and } E \notin \mathcal{H}\}
$$

So, one might expect that Theorem 7.1 generalizes to $d$-uniform properly-connected hypergraphs as follows: $\mathcal{I}(\mathcal{H})$ has a linear resolution if and only if $\mathcal{H}^{c}$ is a triangulated hypergraph. Unfortunately, this is not the case, as shown below, since $\mathcal{H}^{c}$ need not be properly-connected.

Example 7.2 Let $\mathcal{X}=\left\{x_{1}, x_{2}, x_{3}, x_{4}, x_{5}\right\}$. Let $\mathcal{H}=\mathcal{K}_{5}^{3} \backslash\left\{x_{1} x_{2} x_{3}, x_{3} x_{4} x_{5}\right\}$, i.e., $\mathcal{H}$ is the 3-uniform complete hypergraph of order 5 with two edges removed. Then $\mathcal{H}^{c}=$ $\left\{x_{1} x_{2} x_{3}, x_{3} x_{4} x_{5}\right\}$ is not properly-connected, since the two edges intersect at $x_{3}$, but there is no properly-irredundant chain of length 2 between the two edges. Since $\mathcal{H}^{c}$ is not even properly-connected, the notion of a triangulated hypergraph is undefined. However, the ideal $\mathcal{I}(\mathcal{H})$ has the linear resolution

$$
0 \rightarrow R^{4}(-5) \rightarrow R^{11}(-4) \rightarrow R^{8}(-3) \rightarrow \mathcal{I}(\mathcal{H}) \rightarrow 0
$$

We take the first step towards generalizing Theorem 7.1 by asking when $\mathcal{I}(\mathcal{H})$ must have linear first syzygies. Like our previous results, the distance between edges is key.

Definition 7.3 The edge diameter of a $d$-uniform properly-connected hypergraph $\mathcal{H}$ is

$$
\operatorname{diam}(\mathcal{H})=\max \left\{\operatorname{dist}_{\mathcal{H}}(E, H) \mid E, H \in \mathcal{H}\right\},
$$

where the diameter is infinite if there exist two edges not connected by any proper chain.

Since $\mathcal{I}(\mathcal{H})$ is a monomial ideal, we know that its first syzygy module is generated by syzygies $S\left(x^{E}, x^{H}\right)$ for $E, H \in \mathcal{E}$. Moreover, it is clear that $S\left(x^{E}, x^{H}\right)$ is a linear syzygy if and only if $\operatorname{dist}_{\mathcal{H}}(E, H)=1$. We shall see that these syzygies generate all of the syzygies on $\mathcal{I}(\mathcal{H})$ if the diameter of $\mathcal{H}$ is small enough. Indeed, a short enough proper chain will give us a way of writing $S\left(x^{E}, x^{H}\right)$ as a telescoping sum of linear syzygies. The next theorem generalizes [33, Theorem 3.17].

Theorem 7.4 Suppose that $\mathcal{H}$ is a d-uniform properly-connected hypergraph. Then $\mathcal{I}(\mathcal{H})$ has linear first syzygies if and only if $\operatorname{diam}(\mathcal{H}) \leq d$.

Proof Assume first that $\operatorname{diam}(\mathcal{H}) \leq d$. It follows from the Taylor resolution that the first syzygy module of $\mathcal{I}(\mathcal{H})$ is generated by syzygies $S\left(x^{E}, x^{H}\right)$, where $E, H \in \mathcal{E}$. We shall show that $S\left(x^{E}, x^{H}\right)$ is generated by linear syzygies. Let $t=\operatorname{dist}_{\mathcal{H}}(E, H)$. 
Then, since $\operatorname{diam}(\mathcal{H}) \leq d$, we have $t \leq d$. If $\left(E_{0}, \ldots, E_{t}\right)$ is the proper irredundant chain, then by Lemma 4.9 we can write $E=E_{0}=\left\{z_{1}, \ldots, z_{d}\right\}, E_{i}=$ $\left\{y_{1}, \ldots, y_{i}, z_{i+1}, \ldots, z_{d}\right\}$, where $y_{i} \notin E_{j}$ for $j<i$, and $E_{t}=H$.

It can be seen that $S\left(x^{E}, x^{H}\right)$ is given by the equality $y_{1} \cdots y_{t} x^{E_{0}}-z_{1} \cdots z_{t} x^{E_{t}}=$ 0. Furthermore,

$$
y_{1} \ldots y_{t} x^{E_{0}}-z_{1} \ldots z_{t} x^{E_{t}}=\sum_{k=0}^{t-1}\left(\prod_{i=1}^{k} z_{i} \prod_{j=k+2}^{t} y_{j}\right)\left(y_{k+1} x^{E_{k}}-z_{k+1} x^{E_{k+1}}\right) .
$$

Thus, $S\left(x^{E}, x^{H}\right)$ is generated by linear syzygies.

Conversely, suppose that $\mathcal{I}(\mathcal{H})$ has linear first syzygies, that is, $\beta_{1, j}(\mathcal{I}(\mathcal{H}))=0$ for $j \neq d+1$. If $\operatorname{diam}(\mathcal{H}) \geq d+1$, then this implies that there exist at least two edges $E, H$ with $\operatorname{dist}_{\mathcal{H}}(E, H) \geq d+1$, i.e., $\{E, H\}$ is a set of pairwise $(d+1)$-disjoint edges of $\mathcal{H}$. By Theorem 6.5 this implies that $\beta_{1,2 d}(\mathcal{I}(\mathcal{H})) \neq 0$. But this contradicts the fact that $\mathcal{I}(\mathcal{H})$ has linear first syzygies.

Example 7.5 If $\operatorname{diam}(\mathcal{H}) \leq d$ is small, $\mathcal{I}(\mathcal{H})$ may still have nonlinear second syzygies. For example, if $G=C_{5}$ is the 5-cycle, then $\operatorname{diam}(G)=2$. However, $\mathcal{I}(G)=$ $\left(x_{1} x_{2}, x_{2} x_{3}, x_{3} x_{4}, x_{4} x_{5}, x_{5} x_{1}\right)$ has nonlinear second syzygies, since $\beta_{2,5}(\mathcal{I}(G))=1$, as shown in Example 6.10.

Interestingly, if $\mathcal{H}$ is triangulated, knowing that $\mathcal{I}(\mathcal{H})$ has linear first syzygies is enough to know that the entire resolution of $\mathcal{I}(\mathcal{H})$ is linear.

Corollary 7.6 Suppose that $\mathcal{H}$ is a d-uniform properly-connected hypergraph that also is triangulated. Then the following are equivalent:

(a) $\mathcal{I}(\mathcal{H})$ has a linear resolution;

(b) $\mathcal{I}(\mathcal{H})$ has linear first syzygies;

(c) $\operatorname{diam}(\mathcal{H}) \leq d$.

Proof The implication $(a) \Rightarrow(b)$ is immediate, and $(b) \Rightarrow(c)$ is a consequence of Theorem 7.4. To show that $(c) \Rightarrow(a)$, the bound on $\operatorname{diam}(\mathcal{H})$ implies that $\mathcal{H}$ cannot have two or more pairwise $(d+1)$-disjoint edges (otherwise $\operatorname{diam}(\mathcal{H})>d$ ). By Theorem 6.8 this implies that $\operatorname{reg}(\mathcal{I}(\mathcal{H}))=(d-1)+1=d$. Since $\mathcal{I}(\mathcal{H})$ is generated in degree $d$, this forces $\mathcal{I}(\mathcal{H})$ to have a linear resolution.

Restricted to simple graphs, Corollary 7.6 gives the following result.

Corollary 7.7 Suppose that $G$ is a chordal graph. Then the following are equivalent:

(a) $\mathcal{I}(G)$ has a linear resolution;

(b) $\mathcal{I}(G)$ has linear first syzygies;

(c) $\operatorname{diam}(G) \leq 2$. 
Acknowledgements The authors would especially like to thank Jessica Sidman who made some contributions to this paper in its preliminary stages, and with whom we had many useful discussions. The authors would like to thank J. Herzog and X. Zheng for stimulating discussions on the regularity of edge ideals. Part of this research was carried out while the second author visited the first at Tulane University. The authors acknowledge the support from Louisiana Board of Regents for this visit. The second author also thanks Tulane University for its hospitality during his visit. The second author further acknowledges the research support received by NSERC. The computer algebra system COCOA [4] was used to generate examples. We would also like to thank the two anonymous referees for their suggestions and comments.

\section{References}

1. Barile, M. (2006). A note on the edge ideals of Ferrers graphs. Preprint. math.AC/0606353.

2. Berge, C. (1989). Hypergraphs: combinatorics of finite sets. New York: North-Holland.

3. Caboara, M., Faridi, S., \& Selinger, P. (2007). Simplicial cycles and the computation of simplicial trees. Journal of Symbolic Computation, 42, 74-88.

4. CoCoATeam. CoCoA: a system for doing computations in commutative algebra. Available at http: //cocoa.dima.unige.it.

5. Corso, A., \& Nagel, U. (2006). Monomial and toric ideals associated to Ferrers graphs. Preprint. math.AC/0609371.

6. Dirac, G. A. (1961). On rigid circuit graphs. Abhandlungen aus dem Mathematischen Seminar der Universitat Hamburg, 25, 71-76.

7. Eisenbud, D., Green, M., Hulek, K., \& Popescu, S. (2005). Restricting linear syzygies: algebra and geometry. Compositio Mathematica, 141, 1460-1478.

8. Eliahou, S., \& Kervaire, M. (1990). Minimal resolutions of some monomial ideals. Journal of Algebra, $129,1-25$.

9. Eliahou, S., \& Villarreal, R. H. (1999). The second Betti number of an edge ideal. In Aportaciones matematicas, comunicaciones: Vol. 25. XXXI national congress of the Mexican mathematical society (pp. 115-119), Hermosillo, 1998. México: Soc. Mat. Mexicana.

10. Fatabbi, G. (2001). On the resolution of ideals of fat points. Journal of Algebra, 242, 92-108.

11. Faridi, S. (2002). The facet ideal of a simplicial complex. Manuscripta Mathematica, 109, $159-174$.

12. Francisco, C., \& Hà, H. T. (2006, in press). Whiskers and sequentially Cohen-Macaulay graphs. Journal of Combinatorial Theory, Series A. Preprint. math.AC/0605487.

13. Francisco, C., \& Van Tuyl, A. (2007). Sequentially Cohen-Macaulay edge ideals. Proceedings of the American Mathematical Society, 135, 2327-2337.

14. Fröberg, R. (1990). On Stanley-Reisner rings. Topics in algebra, 26(2) 57-70.

15. Herzog, J., Hibi, T., Trung, N. V., \& Zheng, X. (2006). Standard graded vertex cover algebras, cycles, and leaves. Preprint. math.AC/0606357.

16. Herzog, J., Hibi, T., \& Zheng, X. (2004). Monomial ideals whose powers have a linear resolution. Mathematica Scandinavica, 95, 23-32.

17. Herzog, J., Hibi, T., \& Zheng, X. (2006). Cohen-Macaulay chordal graphs. Journal of Combinatorial Theory Series A, 113, 911-916.

18. Herzog, J., Hibi, T., \& Zheng, X. (2004). Dirac's theorem on chordal graphs and Alexander duality. European Journal of Combinatorics, 25, 949-960.

19. Jacques, S. (2004). Betti numbers of graph ideals. University of Sheffield: PhD thesis. math.AC/0410107.

20. Jacques, S., \& Katzman, M. (2005). The Betti numbers of forests. Preprint. math.AC/0401226.

21. Katzman, M. (2006). Characteristic-independence of Betti numbers of graph ideals. Journal of Combinatorial Theory Series A, 113, 435-454.

22. Hà, H. T., \& Van Tuyl, A. (2007). Splittable ideals and the resolutions of monomial ideals. Journal of Algebra, 309, 405-425.

23. Hà, H. T., \& Van Tuyl, A. (2006, in press). Resolutions of squarefree monomial ideals via facet ideals: a survey. Contemporary Mathematics (AMS). Preprint. math.AC/0604301.

24. Miller, E., \& Sturmfels, B. (2004). In Springer GTM: Vol. 227. Combinatorial commutative algebra. Berlin: Springer.

25. Pelsmajer, M. J., Tokaz, J., \& West, D. (2004). New proofs for strongly chordal graphs and chordal bipartite graphs. Preprint. 
26. Roth, M., \& Van Tuyl, A. (2007) On the linear strand of edge ideals. Communications in Algebra, 35, 821-832.

27. Simis, A. (1998). On the Jacobian module associated to a graph. Proceedings of the American Mathematical Society, 126, 989-997.

28. Simis, A., Vasconcelos, W. V., \& Villarreal, R. H. (1994). On the ideal theory of graphs. Journal of Algebra, 167, 389-416.

29. Sturmfels, B., \& Sullivant, S. (2006). Combinatorial secant varieties. Quarterly Journal of Pure and Applied Mathematics, 2, 285-309.

30. Villarreal, R. H. (1995). Rees algebras of edge ideals. Communications in Algebra, 23, 3513-3524.

31. Villarreal, R. H. (1990). Cohen-Macaulay graphs. Manuscripta Mathematica, 66, 277-293.

32. Villarreal, R. H. (2001). Monographs and textbooks in pure and applied mathematics: Vol. 238. Monomial algebras. New York: Dekker.

33. Zheng, X. (2004). Resolutions of facet ideals. Communications in Algebra, 32, 2301-2324. 